Bull. Korean Math. Soc. 49 (2012), No. 4, pp. 829-850

http://dx.doi.org/10.4134/BKMS.2012.49.4.829

\title{
ERROR ESTIMATES OF SEMIDISCRETE DISCONTINUOUS GALERKIN APPROXIMATIONS FOR THE VISCOELASTICITY-TYPE EQUATION
}

\author{
Mi Ray Ohm, Hyun Young Lee, and Jun Yong Shin
}

\begin{abstract}
In this paper, we adopt symmetric interior penalty discontinuous Galerkin (SIPG) methods to approximate the solution of nonlinear viscoelasticity-type equations. We construct finite element space which consists of piecewise continuous polynomials. We introduce an appropriate elliptic-type projection and prove its approximation properties. We construct semidiscrete discontinuous Galerkin approximations and prove the optimal convergence in $L^{2}$ normed space.
\end{abstract}

\section{Introduction}

Let $\Omega$ be an open bounded convex domain in $\mathbb{R}^{d}, d=2,3$ with polygonal/ or polyhedral boundary $\partial \Omega$ and let $0<T<\infty$ be given. In this paper we consider the following nonlinear viscoelasticity-type problems,

$$
\begin{array}{ll}
u_{t t}-\nabla \cdot\left\{a(x, u) \nabla u+b(x, u) \nabla u_{t}\right\}=f(x, u) & \text { in } \Omega \times(0, T] \\
\left(a(x, u) \nabla u+b(x, u) \nabla u_{t}\right) \cdot \mathbf{n}=0 & \text { on } \partial \Omega \times(0, T] \\
u(x, 0)=u_{0}(x), \quad u_{t}(x, 0)=u_{1}(x) & \text { on } \Omega
\end{array}
$$

where $\mathbf{n}$ denotes the unit outward normal vector to $\partial \Omega$ and $u_{0}(x)$ and $u_{1}(x)$ are given functions defined on $\Omega$. The initial data $u_{0}(x), u_{1}(x), a(x, u), b(x, u)$ and $f(x, u)$ are assumed to be such that (1.1) has a sufficiently smooth solution enough to guarantee the regularity conditions appearing in convergence results to be presented below. For the details of the physical significance and various properties of existence and uniqueness of the viscoelasticity-type equations, we refer to $[7,8,10,14,16]$ and references cited there in.

Early Nitsche [11] introduced penalty terms on the boundary of the domain to treat Dirichlet boundary conditions. Douglas and Dupont [5] and Wheeler

Received April 22, 2011; Revised March 21, 2012.

2010 Mathematics Subject Classification. 65M15, 65N30.

Key words and phrases. visoelasticity-type equation, discontinuous Galerkin methods, semidiscrete approximations, $L^{2}$ optimal convergence.

This research was supported by Kyungsung University Research Grants in 2012. 
[17] generalized Nitsche's method and introduced discontinuous Galerkin methods using interior penalties for elliptic and parabolic equations. Darlow et al. [4], and Douglas et al. [6] applied these methods to approximate the behavior of the flow in porous media. These methods which are referred to as interior penalty Galerkin methods are not locally mass conservative. On the other hand, Oden, Babuska and Baumann [12] introduced and analyzed a new type of discontinuous Galerkin method for diffusion problem which was shown to be elementwise conservative. For the polynomials of degree at least 3 and for one dimensional problems, a priori error estimates were proved. Rivière and Wheeler [15] introduced a locally conservative discontinuous Galerkin formulation for nonlinear parabolic equations and derived a priori $L^{\infty}\left(L^{2}\right)$ and $L^{2}\left(H^{1}\right)$ error estimates. However, they achieved suboptimal convergence in $L^{\infty}\left(L^{2}\right)$ norm. Ohm, Lee and Shin [13] constructed a discontinuous Galerkin approximation using interior penalty terms for nonlinear parabolic partial differential equations and proved an optimal $L^{\infty}\left(L^{2}\right)$ error estimate. Compared to the classical Galerkin method, the discontinuous Galerkin method is very well suited for adaptive control of error and can provide high order of accuracy provided that the solution of the model problem is sufficiently smooth.

In [10], Lin and Zhang proved the global $L^{\infty}$-convergence of semidiscrete Galerkin approximation of the solutions to the Sobolev and viscoelasticity type equations using an interpolation postprocessing technique. In this paper we adopt a symmetric discontinuous Galerkin method with interior penalties to construct semidiscrete approximate solutions. We apply symmetric interior penalty discontinuous Galerkin methods to approximate solutions of (1.1) and we obtain the $h p$ convergence in $L^{\infty}\left(L^{2}\right)$ norm. To our knowledge, this paper appears to be the first trial to construct semidiscrete discontinuous Galerkin approximations of viscoelasticity-type equations using symmetric interior penalty method and prove the hp-convergence in $L^{\infty}\left(L^{2}\right)$ norm. This paper is organized as follows. In Section 2 we introduce several notations and preliminaries. In Section 3 we construct finite element spaces and introduce auxiliary projection $\tilde{u}$ of the solution $u$ of (1.1) onto finite element spaces. We prove the projection $\widetilde{u}$ of $u$ converges optimally in $h$ for the $L^{2}$ norm. In Section 4 , we construct semidiscrete discontinuous Galerkin approximations and obtain the $h$-optimal convergence and $p$-suboptimal convergence in $L^{\infty}\left(L^{2}\right)$ norm.

\section{Notations and basis assumptions}

Now we make the following assumptions:

\section{Condition (A)}

there exist positive constants $\underline{k}$ and $\bar{k}$ such that $\underline{k} \leq a(x, y) \leq \bar{k}$ and

Condition (B) $\underline{k} \leq b(x, y) \leq \bar{k}, \forall(x, y) \in \Omega \times \mathbb{R}$,

there exists positive constant $\widetilde{k}$ such that $\left|\frac{\partial a(x, y)}{\partial y}\right| \leq \widetilde{k}$ and $\left|\frac{\partial b(x, y)}{\partial y}\right| \leq$ $\widetilde{k}, \forall(x, y) \in \Omega \times \mathbb{R}$. 
We denote the usual inner product in $L^{2}(\Omega)$ by $(\cdot, \cdot)$ and the norm by $\|\cdot\|$. For an $s \geq 0,1 \leq p \leq \infty$ and $E \subset \mathbb{R}^{d}$ we denote the classical Sobolev spaces by $W^{s, p}(E)$ with norm $\|\cdot\|_{W^{s, p}(E)}$. When $E=\Omega$ we simply write $\|\cdot\|_{W^{s, p}(\Omega)}$ as $\|\cdot\|_{s, p}$ and if $p=2$ we write $\|\cdot\|_{W^{s, p}}$ as $\|\cdot\|_{s}$. And also the usual seminorm of a function defined on $E$ is denoted by $|\cdot|_{s, E}$ and we denote simply $|\cdot|_{s}$ instead of $|\cdot|_{s, \Omega}$ if $E=\Omega$. Since we deal with time dependent problems we need to introduce the norm of a function $v$ mapped from $[0, t]$ to some underlying Banach space $X$, as follows

$$
\|v\|_{L^{p}([0, t] ; X)}=\left(\int_{0}^{t}\|v(\tau)\|_{X}^{p} d \tau\right)^{\frac{1}{p}}, \quad 1 \leq p<\infty
$$

and

$$
\|v\|_{L^{\infty}([0, t] ; X)}=\underset{\tau \in[0, t]}{\operatorname{ess} \sup }\|v(\tau)\|_{X}
$$

We shall abbreviate the notation $L^{p}([0, T] ; X)$ to $L^{p}(X)$.

Let $\mathcal{E}_{h}=\left\{E_{1}, E_{2}, \ldots, E_{N_{h}}\right\}$ be a regular quasi-uniform subdivision of $\Omega$ where $E_{i}$ is a triangle or a quadrilateral if $d=2$ and $E_{i}$ is a 3-simplex or 3 -rectangle if $d=3$. We let $h_{i}=\operatorname{diam}\left(E_{i}\right)$ be the diameter of $E_{i}$ and we let $h=\max \left\{h_{i} \mid 1 \leq i \leq N_{h}\right\}$. We assume that $\mathcal{E}_{h}$ satisfies the following regularity condition: there exists a constant $\alpha>0$ independent of the subdivision such that each $E_{i}$ contains a ball of radius $\alpha h_{i}$. And also we assume that $\mathcal{E}_{h}$ satisfies the following quasiuniformity requirement condition: there is a constant $\gamma>0$ such that $\frac{h}{h_{i}} \leq \gamma, \forall i=1,2, \ldots, N_{h}$.

\section{Finite element spaces and an auxiliary projection}

For an $s \geq 0$ and a given subdivision of $\Omega, \mathcal{E}_{h}=\left\{E_{1}, E_{2}, \ldots, E_{N_{h}}\right\}$, we define the following space

$$
H^{s}\left(\mathcal{E}_{h}\right)=\left\{v \in L^{2}(\Omega)|v|_{E_{i}} \in H^{s}\left(E_{i}\right), \quad i=1,2, \ldots, N_{h}\right\} .
$$

Let the edges of $\mathcal{E}_{h}$ be denoted by $\left\{e_{1}, e_{2}, \ldots, e_{L_{h}}, e_{L_{h}+1}, \ldots, e_{M_{h}}\right\}$ where $e_{k} \subset$ $\Omega, 1 \leq k \leq L_{h}$ and $e_{k} \subset \partial \Omega, L_{h}+1 \leq k \leq M_{h}$. With each edge $e_{k}$, $1 \leq k \leq L_{h}$, we associate a unit outward normal vector $\boldsymbol{n}_{\boldsymbol{k}}$ to $E_{i}$ if $e_{k}=E_{i j}$ where $E_{i j}=\partial E_{i} \cap \partial E_{j}$ and $i<j$. For $L_{h}+1 \leq k \leq M_{h}$, we define $\boldsymbol{n}_{\boldsymbol{k}}=\boldsymbol{n}$ the unit outward normal vector to $\partial \Omega$.

To present the discontinuous Galerkin scheme, we need some functions defined on edges. For $\phi \in H^{s}\left(\mathcal{E}_{h}\right)$ with $s>\frac{1}{2}$, we define the following average function $\{\phi(x)\}$,

$$
\{\phi(x)\}=\frac{1}{2}\left(\left(\left.\phi\right|_{E_{i}}\right)(x)+\left(\left.\phi\right|_{E_{j}}\right)(x)\right), \quad \forall x \in e_{k}, \quad 1 \leq k \leq L_{h}
$$

and jump function $[\phi(x)]$

$$
[\phi(x)]=\left(\left.\phi\right|_{E_{i}}\right)(x)-\left(\left.\phi\right|_{E_{j}}\right)(x), \quad \forall x \in e_{k}, \quad 1 \leq k \leq L_{h},
$$


where $e_{k}=\partial E_{i} \cap \partial E_{j}, i<j$. We associate the following discontinuous norms with the space $H^{s}\left(\mathcal{E}_{h}\right), s \geq 2$

$$
\|\phi\|^{2}=\sum_{i=1}^{N_{h}}\|\phi\|_{0, E_{i}}^{2} \text { and }\|\phi\|_{1}^{2}=\sum_{i=1}^{N_{h}}\left(\|\phi\|_{1, E_{i}}^{2}+h_{i}^{2}\left\|\nabla^{2} \phi\right\|_{0, E_{i}}^{2}\right)+J_{\beta}^{\sigma}(\phi, \phi),
$$

where

$$
J_{\beta}^{\sigma}(\phi, \psi)=\sum_{k=1}^{L_{h}} \frac{\sigma_{k}}{\left|e_{k}\right|^{\beta}} \int_{e_{k}}[\phi][\psi] d s, \quad \beta \geq \frac{1}{2}
$$

is an interior penalty term and each $\sigma_{k}, \forall 1 \leq k \leq L_{h}$ is positive constant. We choose $\left\{\sigma_{k}\right\}_{1 \leq k \leq L_{h}}$ such that $\underset{\sim}{\sigma}<\sigma_{k}<\widetilde{\sigma}, \forall 1 \leq k \leq L_{h}$ holds for some positive constants $\underset{\sim}{\sigma}$ and $\tilde{\sigma}$.

Let $r$ be a positive integer. The finite element space is taken to be

$$
D_{r}\left(\mathcal{E}_{h}\right)=\left\{v \in L^{2}(\Omega):\left.v\right|_{E_{j}} \in P_{r}\left(E_{j}\right), j=1,2, \ldots, N_{h}\right\},
$$

where $P_{r}\left(E_{j}\right)$ denotes the set of polynomials of total degree $\leq r$ on $E_{j}$. Now we state the following trace inequalities proved in [1]. In what follows, we shall denote by $C$ a generic positive constant depending on $\Omega$, the subdivision $\mathcal{E}_{h}$ of $\Omega$, the sobolev norms of $u$ or the constants $\bar{k}, \underline{k}, \widetilde{k}$ but independent of $h$ and $r$, attaining in general different values in different places.

Lemma 3.1. For each $E_{j} \in \mathcal{E}_{h}$, there exists a positive constant $C$ depending only on $\alpha$ and $\gamma$ such that the two following trace inequalities hold:

$$
\begin{aligned}
& \|\phi\|_{L^{2}\left(e_{j}\right)}^{2} \leq C\left(\frac{1}{h_{j}}|\phi|_{0, E_{j}}^{2}+h_{j}|\phi|_{1, E_{j}}^{2}\right), \quad \forall \phi \in H^{1}\left(E_{j}\right), \\
& \left\|\frac{\partial \phi}{\partial \boldsymbol{n}_{j}}\right\|_{L^{2}\left(e_{j}\right)}^{2} \leq C\left(\frac{1}{h_{j}}|\phi|_{1, E_{j}}^{2}+h_{j}|\phi|_{2, E_{j}}^{2}\right), \quad \forall \phi \in H^{2}\left(E_{j}\right),
\end{aligned}
$$

where $e_{j}$ is an edge of $E_{j}$ and $\boldsymbol{n}_{\boldsymbol{j}}$ is the unit outward normal vector to $E_{j}$.

Now we state the following $h p$-approximation properties whose proofs can be found in $[2,3,9]$.

Lemma 3.2. Let $E_{j} \in \mathcal{E}_{h}$ and $\phi \in H^{s}\left(E_{j}\right)$. Then there exist a positive constant $C$ depending on $s, \alpha$ and $\gamma$ but independent of $\phi, r$ and $h$ and $a$ sequence $P_{h} \phi \in P_{r}\left(E_{j}\right), r=1,2, \ldots$, such that for any $0 \leq q \leq s$,

$$
\begin{aligned}
& \left\|\phi-P_{h} \phi\right\|_{W^{q, p}\left(E_{j}\right)} \leq C \frac{h_{j}^{\mu-q}}{r^{s-q}}\|\phi\|_{W^{s, p}\left(E_{j}\right)} \quad s \geq 0, \quad 1 \leq p \leq \infty, \\
& \left\|\phi-P_{h} \phi\right\|_{L^{2}\left(e_{j}\right)} \leq C \frac{h_{j}^{\mu-\frac{1}{2}}}{r^{s-\frac{1}{2}}}\|\phi\|_{s, E_{j}} \quad s>\frac{1}{2}, \\
& \left\|\phi-P_{h} \phi\right\|_{H^{1}\left(e_{j}\right)} \leq C \frac{h_{j}^{\mu-\frac{3}{2}}}{r^{s-\frac{3}{2}}}\|\phi\|_{s, E_{j}} \quad s>\frac{3}{2},
\end{aligned}
$$


where $\mu=\min (r+1, s)$ and $e_{j}$ is an edge or a face of $E_{j}$. Moreover for $e_{k}=E_{i j}$,

$$
\left\|\nabla\left(P_{h} \phi\right)\right\|_{L^{\infty}\left(e_{k}\right)} \leq C\|\nabla \phi\|_{L^{\infty}\left(E_{i} \cup E_{j}\right)} .
$$

Remark. From Lemma 3.2, we may assume that there exists a constant $K^{*}$ such that $\left\|u-P_{h} u\right\|_{L^{\infty}}<K^{*}$ where we choose $K^{*}$ sufficiently large so that $C\left(\left\|u_{0}\right\|_{s}+\|u\|_{L^{\infty}\left(H^{s}\right)}+\|u\|_{L^{\infty}\left(W^{1, \infty}\right)}+\|u\|_{L^{\infty}\left(H^{s}\right)}+\left\|u_{t}\right\|_{L^{\infty}\left(H^{s}\right)}+\left\|u_{t}\right\|_{L^{2}\left(H^{2}\right)}<\right.$ $K^{*}$.

Now we introduce the following bilinear mappings $A(\rho ; \cdot, \cdot), B(\rho ; \cdot, \cdot), A_{t}(\rho ; \cdot, \cdot)$ and $B_{t}(\rho ; \cdot, \cdot)$ defined on $H^{1}\left(\mathcal{E}_{h}\right) \times H^{1}\left(\mathcal{E}_{h}\right)$

$$
\begin{aligned}
A(\rho ; \phi, \psi)= & (a(x, \rho) \nabla \phi, \nabla \psi)-\sum_{k=1}^{L_{h}} \int_{e_{k}}\left\{a(x, \rho) \nabla \phi \cdot \boldsymbol{n}_{\boldsymbol{k}}\right\}[\psi] d s \\
& -\sum_{k=1}^{L_{h}} \int_{e_{k}}\left\{a(x, \rho) \nabla \psi \cdot \boldsymbol{n}_{\boldsymbol{k}}\right\}[\phi] d s+J_{\beta}^{\sigma}(\phi, \psi), \\
B(\rho ; \phi, \psi)= & (b(x, \rho) \nabla \phi, \nabla \psi)-\sum_{k=1}^{L_{h}} \int_{e_{k}}\left\{b(x, \rho) \nabla \phi \cdot \boldsymbol{n}_{\boldsymbol{k}}\right\}[\psi] d s \\
& -\sum_{k=1}^{L_{h}} \int_{e_{k}}\left\{b(x, \rho) \nabla \psi \cdot \boldsymbol{n}_{\boldsymbol{k}}\right\}[\phi] d s+J_{\beta}^{\sigma}(\phi, \psi), \\
A_{t}(\rho ; \phi, \psi)= & \left(\left(\frac{\partial}{\partial t} a(x, \rho)\right) \nabla \phi, \nabla \psi\right)-\sum_{k=1}^{L_{h}} \int_{e_{k}}\left\{\left(\frac{\partial}{\partial t} a(x, \rho)\right) \nabla \phi \cdot \boldsymbol{n}_{\boldsymbol{k}}\right\}[\psi] d s \\
& -\sum_{k=1}^{L_{h}} \int_{e_{k}}\left\{\left(\frac{\partial}{\partial t} a(x, \rho)\right) \nabla \psi \cdot \boldsymbol{n}_{\boldsymbol{k}}\right\}[\phi] d s, \\
& \left(\left(\frac{\partial}{\partial t} b(x, \rho)\right) \nabla \phi, \nabla \psi\right)-\sum_{k=1}^{L_{h}} \int_{e_{k}}\left\{\left(\frac{\partial}{\partial t} b(x, \rho)\right) \nabla \phi \cdot \boldsymbol{n}_{\boldsymbol{k}}\right\}[\psi] d s \\
& -\sum_{k=1}^{L_{h}} \int_{e_{k}}\left\{\left(\frac{\partial}{\partial t} b(x, \rho)\right) \nabla \psi \cdot \boldsymbol{n}_{\boldsymbol{k}}\right\}[\phi] d s .
\end{aligned}
$$

And we define the following weak formulation of the problem (1.1): Find $u \in H^{1}\left(\mathcal{E}_{h}\right)$ such that

$$
\left(u_{t t}, v\right)+A(u ; u, v)+B\left(u ; u_{t}, v\right)=(f(x, u), v), \quad \forall v \in H^{1}\left(\mathcal{E}_{h}\right) .
$$

For a $\lambda>0$ we define the following bilinear forms $A_{\lambda}(\rho ; \cdot, \cdot)$ and $B_{\lambda}(\rho ; \cdot, \cdot)$ on $H^{1}\left(\mathcal{E}_{h}\right) \times H^{1}\left(\mathcal{E}_{h}\right)$ by

$$
\begin{aligned}
& A_{\lambda}(\rho ; \phi, \psi)=A(\rho ; \phi, \psi)+\lambda(\phi, \psi), \\
& B_{\lambda}(\rho ; \phi, \psi)=B(\rho ; \phi, \psi)+\lambda(\phi, \psi) .
\end{aligned}
$$


Then $A_{\lambda}$ and $B_{\lambda}$ satisfy the following boundedness and coercivity properties. The proof of Lemmas 3.4 and 3.4 with $d=1,2$ are given in [13]. For $d \geq 1$, the proofs can be obtained similarly, but for the completeness of description we provide the proof of Lemma 3.3. Lemma 3.4 can be proved similarly so we omit the proof.

Lemma 3.3. For $a \lambda>0$ and $a \beta \geq \frac{1}{d-1}$ if the functions $a(x, y)$ and $b(x, y)$ satisfy Condition $(A)$, then there exists a constant $C>0$ independent of $h$ and $r$ satisfying

$$
\begin{aligned}
& \left|A_{\lambda}(\rho ; \phi, \psi)\right| \leq C\|\phi\|_{1}\|\psi\|_{1}, \quad \forall \phi, \psi \in H^{2}\left(\mathcal{E}_{h}\right), \\
& \left|B_{\lambda}(\rho ; \phi, \psi)\right| \leq C\|\phi\|_{1}\|\psi\|_{1}, \quad \forall \phi, \psi \in H^{2}\left(\mathcal{E}_{h}\right) .
\end{aligned}
$$

Proof. Let $\phi, \psi \in H^{2}\left(\mathcal{E}_{h}\right)$. From the definition of $A_{\lambda}(\rho, \phi, \psi)$, we have

$$
\begin{aligned}
\left|A_{\lambda}(\rho ; \phi, \psi)\right| \leq & |(a(x, \rho) \nabla \phi, \nabla \psi)|+\left|\sum_{k=1}^{L_{h}} \int_{e_{k}}\left\{a(x, \rho) \nabla \phi \cdot \boldsymbol{n}_{\boldsymbol{k}}\right\}[\psi] d s\right| \\
& +\left|\sum_{k=1}^{L_{h}} \int_{e_{k}}\left\{a(x, \rho) \nabla \psi \cdot \boldsymbol{n}_{\boldsymbol{k}}\right\}[\phi] d s\right|+\left|J_{\beta}^{\sigma}(\phi, \psi)\right|+\lambda|(\phi, \psi)| \\
= & E_{1}+E_{2}+E_{3}+E_{4}+E_{5} .
\end{aligned}
$$

By Condition (A), we have $E_{1} \leq \bar{k}\|\phi\|_{1}\|\psi\|_{1}$. Applying Lemma 3.1 and the condition $\beta \geq \frac{1}{d-1}$, we estimate $E_{2}$ as follows

$$
\begin{aligned}
E_{2} & \leq \bar{k} \sum_{k=1}^{L_{h}} \int_{e_{k}}\left|\left\{\nabla \phi \cdot \boldsymbol{n}_{\boldsymbol{k}}\right\}\right||[\psi]| d s \\
& \leq \bar{k}\left(\sum_{k=1}^{L_{h}} \frac{\left|e_{k}\right|^{\beta}}{\sigma_{k}} \int_{e_{k}}\left\{\nabla \phi \cdot \boldsymbol{n}_{\boldsymbol{k}}\right\}^{2} d s\right)^{\frac{1}{2}}\left(\sum_{k=1}^{L_{h}} \frac{\sigma_{k}}{\left|e_{k}\right|^{\beta}} \int_{e_{k}}[\psi]^{2} d s\right)^{\frac{1}{2}} \\
& \leq C\left(\sum_{i=1}^{N_{h}} \frac{\left(h^{d-1}\right)^{\beta}}{\underset{\sim}{\sigma}}\left[\frac{1}{h}|\phi|_{1, E_{i}}^{2}+h|\phi|_{2, E_{i}}^{2}\right]\right)^{\frac{1}{2}}\|\psi\|_{1} \\
& \leq C\|\phi\|_{1}\|\psi\| \|_{1} .
\end{aligned}
$$

Similarly $E_{3} \leq C\|\phi\|_{1}\|\psi\|_{1}$ can be obtained. By the definition of $J_{\beta}^{\sigma}(\phi, \psi)$, we have

$$
\begin{aligned}
\left|J_{\beta}^{\sigma}(\phi, \psi)\right| & =\left|\sum_{k=1}^{P_{h}} \frac{\sigma_{k}}{\left|e_{k}\right|^{\beta}} \int[\phi][\psi] d s\right| \\
& \leq\left|\sum_{k=1}^{P_{h}} \frac{\sigma_{k}}{\left|e_{k}\right|^{\beta}}\left(\int_{e_{k}}[\phi]^{2} d s\right)^{\frac{1}{2}}\left(\int_{e_{k}}[\psi]^{2} d s\right)^{\frac{1}{2}}\right| \\
& \leq\left(\sum_{k=1}^{P_{h}} \frac{\sigma_{k}}{\left|e_{k}\right|^{\beta}} \int_{e_{k}}[\phi]^{2} d s\right)^{\frac{1}{2}}\left(\sum_{k=1}^{P_{h}} \frac{\sigma_{k}}{\left|e_{k}\right|^{\beta}} \int_{e_{k}}[\psi]^{2} d s\right)^{\frac{1}{2}}
\end{aligned}
$$




\section{$=\|\phi\|_{1}\|\psi\|_{1}$.}

Trivially $E_{5} \leq \lambda\|\phi\|_{1}\|\psi\|_{1}$ holds. Therefore we have $\left|A_{\lambda}(\rho ; \phi, \psi)\right| \leq C\|\phi\|_{1}\|\psi\|_{1}$. By the similar method the boundedness result for $B_{\lambda}(\rho ; \phi, \psi)$ can be proved.

Lemma 3.4. For $a \lambda>0, a \beta \geq \frac{1}{d-1}$ and a sufficiently large $\sigma$ if the functions $a(x, y)$ and $b(x, y)$ satisfy Condition $(A)$, then there exists a constant $\widetilde{c}>0$ independent of $h$ and $r$ satisfying

$$
\begin{array}{ll}
A_{\lambda}(\rho ; \phi, \phi) \geq \widetilde{c}\|\phi\|_{1}^{2}, & \forall \phi \in D_{r}\left(\mathcal{E}_{h}\right), \\
B_{\lambda}(\rho ; \phi, \phi) \geq \widetilde{c}\|\phi\|_{1}^{2}, & \forall \phi \in D_{r}\left(\mathcal{E}_{h}\right) .
\end{array}
$$

The results of Lemma 3.3 and Lemma 3.4 provide the existence and uniqueness of the following auxiliary projection $\widetilde{u}(x, t) \in D_{r}\left(\varepsilon_{h}\right)$ of $u(x, t)$ : Find $\widetilde{u}(t):[0, T] \rightarrow D_{r}\left(\mathcal{E}_{h}\right)$ such that

$$
\begin{cases}A_{\lambda}(u ; u-\widetilde{u}, v)+B_{\lambda}\left(u ; u_{t}-\widetilde{u}_{t}, v\right)=0, & \forall v \in D_{r}\left(\mathcal{E}_{h}\right), \quad \forall t>0 \\ \widetilde{u}(x, 0)=P_{h}\left(u_{0}(x)\right), \quad \widetilde{u}_{t}(x, 0)=P_{h}\left(u_{1}(x)\right) & \forall x \in \Omega\end{cases}
$$

where $P_{h}\left(u_{0}(x)\right)$ and $P_{h}\left(u_{1}(x)\right)$ are the projections of $u_{0}(x)$ and $u_{1}(x)$, respectively generated by Lemma 3.2. Now we construct the semidiscrete discontinuous Galerkin approximations as follows: Find $U(\cdot, t) \in D_{r}\left(\mathcal{E}_{h}\right), \forall t \in(0, T]$ such that

$$
\begin{cases}\left(U_{t t}, v\right)+A(U ; U, v)+B\left(U ; U_{t}, v\right)=(f(x, U), v), & \forall v \in D_{r}\left(\mathcal{E}_{h}\right), \\ U(x, 0)=\widetilde{u}(x, 0) & \forall x \in \Omega, \\ U_{t}(x, 0)=\widetilde{u}_{t}(x, 0) & \forall x \in \Omega\end{cases}
$$

To proceed the convergence of the semidiscrete approximation $U(x, t)$ to $u(x, t)$, we let $\eta(x, t)=u(x, t)-\widetilde{u}(x, t), \theta(x, t)=P_{h} u(x, t)-\widetilde{u}(x, t)$ and $\xi(x, t)=$ $\widetilde{u}(x, t)-U(x, t)$. We let $\widetilde{H}(\Omega)=\left\{\psi \in H^{1}(\Omega) \mid \nabla \psi \cdot \boldsymbol{n}=0\right.$ on $\left.\partial \Omega\right\}$. By the definition of $A_{\lambda}, B_{\lambda}, A_{t}$ and $B_{t}$ we have the following Lemma 3.5.

Lemma 3.5. Suppose that $a(x, y)$ and $b(x, y)$ satisfy Conditions $(A)$ and $(B)$, then there exists a constant $C>0$ such that

$$
\begin{aligned}
& \left|A_{\lambda}(u ; \eta, \psi)\right| \leq C\|\eta\|\|\| \psi \|_{2}, \\
& \left|B_{\lambda}(u ; \eta, \psi)\right| \leq C\|\eta\|\|\psi\| \|_{2}, \\
& \left|A_{t}(u ; \eta, \psi)\right| \leq C\|\eta\|\|\psi\|_{2}, \\
& \left|B_{t}(u ; \eta, \psi)\right| \leq C\|\eta\|\|\psi\|_{2},
\end{aligned}
$$

hold for $\psi \in H^{2}(\Omega) \cap \widetilde{H}(\Omega)$.

Proof. By the definition of $A_{\lambda}$ and the continuity of $\psi$ we have

$$
A_{\lambda}(u ; \eta, \psi)=(a(x, u) \nabla \eta, \nabla \psi)-\sum_{k=1}^{L_{h}} \int_{e_{k}}\left\{a(x, u) \nabla \eta \cdot \boldsymbol{n}_{\boldsymbol{k}}\right\}[\psi] d s
$$




$$
\begin{aligned}
& -\sum_{k=1}^{L_{h}} \int_{e_{k}}\left\{a(x, u) \nabla \psi \cdot \boldsymbol{n}_{\boldsymbol{k}}\right\}[\eta] d s+J_{\beta}^{\sigma}(\eta, \psi)+\lambda(\eta, \psi) \\
= & (a(x, u) \nabla \eta, \nabla \psi)-\sum_{k=1}^{L_{h}} \int_{e_{k}}\left\{a(x, u) \nabla \psi \cdot \boldsymbol{n}_{\boldsymbol{k}}\right\}[\eta] d s+\lambda(\eta, \psi) .
\end{aligned}
$$

On the other hand, by the continuity of $\nabla \psi \cdot \boldsymbol{n}_{\boldsymbol{k}}$ across interior edge $e_{k}$ and $\nabla \psi \cdot \boldsymbol{n}=0$ on $\partial \Omega$, the following holds:

$$
\begin{aligned}
(a(x, u) \eta, \Delta \psi)= & \sum_{j=1}^{N_{h}} \int_{E_{j}}(a(x, u) \eta) \Delta \psi d x \\
= & \sum_{j=1}^{N_{h}}\left[\int_{\partial E_{j}} a(x, u) \eta \nabla \psi \cdot \boldsymbol{n}_{j} d s-\int_{E_{j}} \nabla(a(x, u) \eta) \cdot \nabla \psi d x\right] \\
= & -(a(x, u) \nabla \eta, \nabla \psi)-\sum_{j=1}^{N_{h}} \int_{E_{j}} \nabla(a(x, u)) \eta \cdot \nabla \psi d x \\
& +\int_{\partial \Omega} a(x, u) \eta \nabla \psi \cdot \boldsymbol{n} d s+\sum_{k=1}^{L_{h}} \int_{e_{k}}\left\{\nabla \psi \cdot \boldsymbol{n}_{\boldsymbol{k}}\right\}[a(x, u) \eta] d s \\
= & -(a(x, u) \nabla \eta, \nabla \psi)-\sum_{j=1}^{N_{h}} \int_{E_{j}} \nabla(a(x, u)) \eta \cdot \nabla \psi d x \\
& +\sum_{k=1}^{L_{h}} \int_{e_{k}}\left\{\nabla \psi \cdot \boldsymbol{n}_{\boldsymbol{k}}\right\}[a(x, u) \eta] d s .
\end{aligned}
$$

Therefore

$$
A_{\lambda}(u ; \eta, \psi)=-(a(x, u) \eta, \Delta \psi)-\sum_{j=1}^{N_{h}} \int_{E_{j}} \nabla(a(x, u)) \eta \cdot \nabla \psi d x+\lambda(\eta, \psi),
$$

from which we get, $\left|A_{\lambda}(u ; \eta, \psi)\right| \leq C\|\eta\|\|\psi\|_{2}$. Similarly we can obtain the results for $B_{\lambda}, A_{t}$ and $B_{t}$.

As shown in [13] to prove the following lemma we need the regularity property of the elliptic operator $L(u) w=\nabla \cdot(a(x, u) \nabla w)+\lambda w$ with $u$, the solution of the problem (1.1). The regularity result of the preceding elliptic operator can be sufficiently obtained when $\Omega$ is a bounded open convex domain. We state the following lemma whose proof can be found in [13].

Lemma 3.6. Suppose that $F: H^{2}\left(\mathcal{E}_{h}\right) \rightarrow \mathbb{R}$ is a linear function and that there exists $\phi \in H^{2}\left(\mathcal{E}_{h}\right)$ satisfying $B_{\lambda}(u ; \phi, v)=F(v), \forall v \in D_{r}\left(\mathcal{E}_{h}\right)$. If there exist positive constants $M_{1}$ and $M_{2}$ satisfying

$$
|F(\omega)| \leq M_{1}\|\omega\|_{1}, \quad \omega \in H^{2}\left(\mathcal{E}_{h}\right),
$$




$$
|F(\psi)| \leq M_{2}\|\psi\|_{2}, \quad \psi \in H^{2}(\Omega) \cap \widetilde{H}(\Omega),
$$

then the following estimation holds

$$
\|\phi\| \leq C\left(\|\phi\|_{1}+M_{1}\right) h+M_{2} .
$$

Now we obtain the following approximation properties for $\eta$ whose proofs can be found in [13]. Hereafter we omit the time variable $t$ if there is no possibility of confusion. In the remaining part of this paper we assume that $a(x, y)$ and $b(x, y)$ satisfy Conditions $(\mathrm{A})$ and $(\mathrm{B})$.

Theorem 3.1. If $u_{0} \in H^{s}, u_{t} \in L^{2}\left(H^{s}\right)$ and $\beta=\frac{1}{d-1}$, then there exists a constant $C>0$ independent of $h$ and $r$ satisfying

(i) $\left\|\eta_{t}(t)\right\|+h\left\|\eta_{t}(t)\right\|_{1} \leq C \frac{h^{\mu}}{r^{s-2}}\left(\left\|u_{0}\right\|_{s}+\left\|u_{t}\right\|_{s}+\left\|u_{t}\right\|_{L^{2}\left(H^{s}\right)}\right)$,

(ii) $\|\eta(t)\|+h\|\eta(t)\|_{1} \leq C \frac{h^{\mu}}{r^{s-2}}\left(\left\|u_{0}\right\|_{s}+\left\|u_{t}\right\|_{L^{2}\left(H^{s}\right)}\right)$,

where $\mu=\min (r+1, s), r \geq \frac{d}{2}$ and $s \geq 1+\frac{d}{2}$.

Theorem 3.2. If $u_{0} \in H^{s}, u_{t} \in L^{2}\left(H^{s}\right), u_{t t}(t) \in H^{s}$ and $\beta=\frac{1}{d-1}$, then there exists a constant $C$ independent of $h$ and $r$ such that

(i) $\left\|\eta_{t t}(t)\right\|_{1} \leq C \frac{h^{\mu-1}}{r^{s-2}}\left(\left\|u_{0}\right\|_{s}+\left\|u_{t}\right\|_{s}+\left\|u_{t}\right\|_{L^{2}\left(H^{s}\right)}+\left\|u_{t t}(t)\right\|_{s}\right)$,

(ii) $\left\|\eta_{t t}(t)\right\| \leq C \frac{h^{\mu}}{r^{s-2}}\left(\left\|u_{0}\right\|_{s}+\left\|u_{t}\right\|_{s}+\left\|u_{t}\right\|_{L^{2}\left(H^{s}\right)}+\left\|u_{t t}(t)\right\|_{s}\right)$, where $\mu=\min (r+1, s), r \geq \frac{d}{2}$ and $s \geq 1+\frac{d}{2}$.

Proof. (i) Differentiating both sides of (3.2) with respect to $t$, we get

$$
\begin{aligned}
& \left(\left(\frac{\partial}{\partial t} a(x, u)\right) \nabla \eta, \nabla v\right)-\sum_{k=1}^{L_{h}} \int_{e_{k}}\left\{\left(\frac{\partial}{\partial t} a(x, u)\right) \nabla \eta \cdot \boldsymbol{n}_{\boldsymbol{k}}\right\}[v] d s \\
& -\sum_{k=1}^{L_{h}} \int_{e_{k}}\left\{\left(\frac{\partial}{\partial t} a(x, u)\right) \nabla v \cdot \boldsymbol{n}_{\boldsymbol{k}}\right\}[\eta] d s+A_{\lambda}\left(u ; \eta_{t}, v\right)+\left(\left(\frac{\partial}{\partial t} b(u)\right) \nabla \eta_{t}, \nabla v\right) \\
& -\sum_{k=1}^{L_{h}} \int_{e_{k}}\left\{\left(\frac{\partial}{\partial t} b(x, u)\right) \nabla \eta_{t} \cdot \boldsymbol{n}_{\boldsymbol{k}}\right\}[v] d s-\sum_{k=1}^{L_{h}} \int_{e_{k}}\left\{\left(\frac{\partial}{\partial t} b(x, u)\right) \nabla v \cdot \boldsymbol{n}_{\boldsymbol{k}}\right\}\left[\eta_{t}\right] d s \\
& +B_{\lambda}\left(u ; \eta_{t t}, v\right)=0 .
\end{aligned}
$$

By adopting the definitions of $A_{t}$ and $B_{t}$ to (3.4), we get

(3.5) $\quad B_{\lambda}\left(u ; \eta_{t t}, v\right)=-A_{\lambda}\left(u ; \eta_{t}, v\right)-A_{t}(u ; \eta, v)-B_{t}\left(u ; \eta_{t}, v\right), \quad \forall v \in D_{r}\left(\mathcal{E}_{h}\right)$.

Then by (3.5) we have

$$
\begin{aligned}
B_{\lambda}\left(u ; \theta_{t t}, \theta_{t t}\right)= & B_{\lambda}\left(u ; u_{t t}-\widetilde{u}_{t t}-u_{t t}+P_{h} u_{t t}, \theta_{t t}\right) \\
= & B_{\lambda}\left(u ; \eta_{t t}, \theta_{t t}\right)-B_{\lambda}\left(u ; u_{t t}-P_{h} u_{t t}, \theta_{t t}\right) \\
= & -A_{\lambda}\left(u ; \eta_{t}, \theta_{t t}\right)-A_{t}\left(u ; \eta, \theta_{t t}\right)-B_{t}\left(u ; \eta_{t}, \theta_{t t}\right) \\
& -B_{\lambda}\left(u ; u_{t t}-P_{h} u_{t t}, \theta_{t t}\right) .
\end{aligned}
$$

By Lemma 3.3 and Lemma 3.4, we obtain

$$
\left\|\theta_{t t}\right\|_{1} \leq C\left(\left\|\eta_{t}\right\|_{1}+\|\eta\|_{1}+\left\|u_{t t}-P_{h} u_{t t}\right\|_{1}\right)
$$


which implies that

$$
\begin{aligned}
\left\|\eta_{t t}\right\|_{1} & \leq\left\|u_{t t}-P_{h} u_{t t}\right\|_{1}+\left\|P_{h} u_{t t}-\widetilde{u}_{t t}\right\|_{1} \\
& \leq\left\|u_{t t}-P_{h} u_{t t}\right\|_{1}+C\left(\left\|\eta_{t}\right\|_{1}+\|\eta\|_{1}+\left\|u_{t t}-P_{h} u_{t t}\right\|_{1}\right) .
\end{aligned}
$$

From Lemma 3.1 we have the following estimation

$$
\begin{aligned}
& =\sum_{i=1}^{N_{h}}\left\|u-P_{h} u\right\|_{1, E_{i}}^{2}+\sum_{i=1}^{N_{h}} h_{i}^{2}\left\|\nabla^{2}\left(u-P_{h} u\right)\right\|_{0, E_{i}}^{2}+J_{\beta}^{\sigma}\left(u-P_{h} u, u-P_{h} u\right) \\
& \leq C\left(\frac{h^{2(\mu-1)}}{r^{2(s-2)}}\|u\|_{s}^{2}+h^{-(d-1) \beta} \sum_{k=1}^{L_{h}}\left\|\left[u-P_{h} u\right]\right\|_{0, e_{k}}^{2}\right) \\
& \leq C\left[\frac{h^{2(\mu-1)}}{r^{2(s-2)}}\|u\|_{s}^{2}+\frac{h^{-(d-1) \beta-1}}{r^{2(s-1)}}\left(h^{2 \mu}\|u\|_{s}^{2}\right)\right] \\
& \leq C \frac{h^{2(\mu-1)}}{r^{2(s-2)}}\|u\|_{s}^{2} .
\end{aligned}
$$

By applying the inequality above with $u_{t t}$ to (3.6) we obtain the following

$$
\begin{aligned}
\left\|\eta_{t t}\right\|_{1}^{2} & \leq C\left(\left\|u_{t t}-P_{h} u_{t t}\right\|_{1}^{2}+\|\eta\|_{1}^{2}+\left\|\eta_{t}\right\|_{1}^{2}\right) \\
& \leq C \frac{h^{2(\mu-1)}}{r^{2(s-2)}}\left(\left\|u_{0}\right\|_{s}^{2}+\left\|u_{t}\right\|_{s}^{2}+\left\|u_{t}\right\|_{L^{2}\left(H^{s}\right)}^{2}+\left\|u_{t t}\right\|_{s}^{2}\right),
\end{aligned}
$$

which implies

$$
\left\|\eta_{t t}\right\|_{1} \leq C \frac{h^{\mu-1}}{r^{s-2}}\left(\left\|u_{0}\right\|_{s}+\left\|u_{t}\right\|_{s}+\left\|u_{t}\right\|_{L^{2}\left(H^{s}\right)}+\left\|u_{t t}\right\|_{s}\right) .
$$

(ii) By Lemma 3.3 there exists a constant $C>0$ satisfying

$$
\left|B_{\lambda}\left(u ; \eta_{t t}, v\right)\right| \leq C\left\|\eta_{t t}\right\|_{1}\|v\|_{1}, \quad \forall v \in H^{2}\left(\mathcal{E}_{h}\right) .
$$

By applying the definitions of $A_{t}$ and $B_{t}$ and Lemma 3.5 we have the following estimation

$$
\left|A_{\lambda}\left(u ; \eta_{t}, v\right)\right|+\left|A_{t}(u ; \eta, v)\right|+\left|B_{t}\left(u ; \eta_{t}, v\right)\right| \leq M_{2}\|v\|_{2} \quad \forall v \in H^{2}(\Omega) \cap \widetilde{H}(\Omega)
$$

with $M_{2}=C\left(\left\|\eta_{t}\right\|+\|\eta\|\right)$. By applying Lemma 3.6 to (3.5) with $M_{1}=C\left\|\eta_{t t}\right\|_{1}$, we have

$$
\begin{aligned}
\left\|\eta_{t t}\right\| & \leq C\left(h\left\|\eta_{t t}\right\|_{1}+\left\|\eta_{t}\right\|+\|\eta\|\right) \\
& \leq C \frac{h^{\mu}}{r^{s-2}}\left(\left\|u_{0}\right\|_{s}+\left\|u_{t}\right\|_{s}+\left\|u_{t}\right\|_{L^{2}\left(H^{s}\right)}+\left\|u_{t t}\right\|_{s}\right) .
\end{aligned}
$$

\section{The convergence of semidiscrete discontinuous Galerkin approximations}

Before we prove the convergence of the semidiscrete approximation defined in (3.3), we will show the existence and uniqueness of semidiscrete approximation in the following theorem. 
Theorem 4.1. The following statements hold:

(i) If $f$ is a continuous function, then there exists a semidiscrete discontinuous Galerkin approximation $U(x, t)$ satisfying (3.3). Furthermore $\|U(t)\|$ and $\left\|U_{t}(t)\right\|$ are continuous with respect to $t$.

(ii) In addition to the hypothesis of (i), if $f$ is globally Lipschitz continuous, i.e., there exists a constant $L>0$ such that $|f(x, u)-f(x, v)| \leq$ $L|u-v|, \forall(x, u),(x, v) \in \Omega \times \mathbb{R}$, then (3.3) has a unique semidiscrete approximation $U(x, t)$.

Proof. Let $\left\{\phi_{i}(x)\right\}_{i=1}^{m}$ be a basis of $D_{r}\left(\mathcal{E}_{h}\right)$ and $U(x, t)=\sum_{i=1}^{m} \alpha_{i}(t) \phi_{i}(x)$. Then (3.3) reduces to a system of nonlinear ordinary differential equations such that

$$
\begin{aligned}
& \left(\sum_{i=1}^{m} \alpha_{i}^{\prime \prime}(t) \phi_{i}(x), \phi_{j}(x)\right)+A\left(U ; \sum_{i=1}^{m} \alpha_{i}(t) \phi_{i}(x), \phi_{j}(x)\right) \\
& +B\left(U ; \sum_{i=1}^{m} \alpha_{i}^{\prime}(t) \phi_{i}(x), \phi_{j}(x)\right)=\left(f\left(x, \sum_{i=1}^{m} \alpha_{i}(t) \phi_{i}(x)\right), \phi_{j}(x)\right), 1 \leq j \leq m .
\end{aligned}
$$

which implies the following initial value problem

$$
\left\{\begin{array}{l}
\mathbf{S} \boldsymbol{\alpha}^{\prime \prime}(t)=-\mathbf{T}(\boldsymbol{\alpha}) \boldsymbol{\alpha}^{\prime}(t)-\mathbf{W}(\boldsymbol{\alpha}) \boldsymbol{\alpha}+\mathbf{F}(\alpha), \\
\boldsymbol{\alpha}(0)=\boldsymbol{\alpha}_{0}, \quad \boldsymbol{\alpha}^{\prime}(0)=\boldsymbol{\alpha}_{1}
\end{array}\right.
$$

where $\boldsymbol{\alpha}=\left(\alpha_{1}(t), \alpha_{2}(t), \ldots, \alpha_{m}(t)\right)^{T}, \boldsymbol{\alpha}_{0}$ and $\boldsymbol{\alpha}_{1}$ are initial value vectors, $\mathbf{S}=$ $\left(S_{i j}\right)_{1 \leq i, j \leq m}, \boldsymbol{T}(\boldsymbol{\alpha})=\left(T_{i j}(\boldsymbol{\alpha})\right)_{1 \leq i, j \leq m}$ and $\boldsymbol{W}(\boldsymbol{\alpha})=\left(W_{i j}(\boldsymbol{\alpha})\right)_{1 \leq i, j \leq m}$ are $m \times$ $m$ symmetric matrices and $\boldsymbol{F}(\boldsymbol{\alpha})=\left(F_{1}(\boldsymbol{\alpha}), F_{2}(\boldsymbol{\alpha}), \ldots, F_{m}(\boldsymbol{\alpha})\right)^{T}$ is a vector. Matrices $S, T$ and $W$ are defined by $S_{i j}=\left(\phi_{i}, \phi_{j}\right), T_{i j}=B\left(U ; \phi_{i}, \phi_{j}\right), W_{i j}=$ $A\left(U ; \phi_{i}, \phi_{j}\right)$ and $F_{j}=\left(f(x, U), \phi_{j}\right)$.

Let $\boldsymbol{\beta}(t)=\left(\alpha_{1}^{\prime}(t), \alpha_{2}^{\prime}(t), \ldots, \alpha_{m}^{\prime}(t)\right)^{T}$ and let $\widetilde{\boldsymbol{S}}$ be the $2 m$ by $2 m$ block diagonal matrix with $\boldsymbol{I}, \boldsymbol{S}$ as block diagonal elements. $P_{h}\left(u_{0}(x)\right)=U(x, 0)$ and $P_{h}\left(u_{1}(x)\right)=U_{t}(x, 0)$ can be represented by

$$
U(x, 0)=\sum_{i=1}^{m} \alpha_{i}(0) \phi_{i}(x) \text { and } U_{t}(x, 0)=\sum_{i=1}^{m} \beta_{i}(0) \phi_{i}(x) .
$$

Then (4.2) can be reduced to initial boundary value problem associated with a system of 1 st order differential equations.

$$
\left(\begin{array}{ll}
\mathbf{I} & 0 \\
0 & \mathbf{S}
\end{array}\right)\left(\begin{array}{c}
\frac{d \boldsymbol{\alpha}}{d t} \\
\frac{d \boldsymbol{\beta}}{d t}
\end{array}\right)=\left(\begin{array}{c}
\boldsymbol{\beta}(t) \\
-\boldsymbol{T}(\boldsymbol{\alpha}) \boldsymbol{\beta}(t)-\boldsymbol{W}(\boldsymbol{\alpha}) \boldsymbol{\alpha}(t)+\boldsymbol{F}(\boldsymbol{\alpha})
\end{array}\right),
$$

with initial condition $(\boldsymbol{\alpha}(0), \boldsymbol{\beta}(0))^{T}=\left(\alpha_{1}(0), \ldots, \alpha_{m}(0), \beta_{1}(0), \ldots, \beta_{m}(0)\right)^{T}$. For $\mathbf{v}=\left(v_{1}, v_{2}, \ldots, v_{m}\right)^{T} \in \mathbb{R}^{m}$ and $\mathbf{y}=\left(y_{1}, y_{2}, \ldots, y_{m}\right)^{T} \in \mathbb{R}^{m}$ we let

$$
v(x)=\sum_{i=1}^{m} v_{i} \phi_{i}(x) \text { and } y(x)=\sum_{i=1}^{m} y_{i} \phi_{i}(x) .
$$


Then we get

$$
\left(\mathbf{v}^{T}, \mathbf{y}^{T}\right) \widetilde{\boldsymbol{S}}\left(\begin{array}{l}
\mathbf{v} \\
\mathbf{y}
\end{array}\right)=\sum_{i=1}^{m} v_{i}^{2}+\sum_{i=1}^{m} y_{i} S_{i j} y_{j}=\sum_{i=1}^{m} v_{i}^{2}+(y, y) \geq 0 .
$$

Therefore $\widetilde{\boldsymbol{S}}$ is positive definite. By the continuity condition on $f$, and the theory of ordinary differential equations, $\boldsymbol{\alpha}(t)$ and $\boldsymbol{\beta}(t)$ exist which completes the proof of the existence of the semidiscrete approximation. This result suffices to show that $\alpha_{i}(t)$ and $\beta_{i}(t)$ are continuous with respect to $t$ so that $\|U(t)\|$ and $\left\|U_{t}(t)\right\|$ are also continuous which completes the proof of the statement (i). If the functions $a$ and $b$ are bounded and $f$ is Lipschtz continuous then there exists a unique pair of $\{\boldsymbol{\alpha}(t), \boldsymbol{\beta}(t)\}$ by the theory of ordinary differential equation. This completes the proof of (ii).

Lemma 4.1. Suppose that $u_{0} \in H^{s}, u(t) \in H^{s}$ and $u_{t} \in L^{2}\left(H^{s}\right)$ with $s \geq \frac{d}{2}+1$. For $a \lambda>0$ and $\beta=1 /(d-1)$, there exists a constant $C$ satisfying

$$
\|\theta(t)\|_{1} \leq C\left(\frac{h^{\mu-1}}{r^{s-2}}\right)\left(\left\|u_{0}\right\|_{s}+\|u\|_{s}+\left\|u_{t}\right\|_{s}+\left\|u_{t}\right\|_{L^{2}\left(H^{s}\right)}\right)
$$

and

$$
\left\|\theta_{t}(t)\right\|_{1} \leq c\left(\frac{h^{\mu-1}}{r^{s-2}}\right)\left(\left\|u_{0}\right\|_{s}+\left\|u_{t}\right\|_{s}+\left\|u_{t}\right\|_{L^{2}\left(H^{s}\right)}\right),
$$

where $\mu=\min (r+1, s)$ and $r \geq d / 2$.

Proof. By Lemma 3.4 and Lemma 3.3 we obtain

$$
\begin{aligned}
\|\theta\|_{1}^{2} & \leq C A_{\lambda}(u ; \theta, \theta)=C\left(A_{\lambda}(u ; \theta, \theta)-A_{\lambda}(u ; \eta, \theta)-B_{\lambda}\left(u ; \eta_{t}, \theta\right)\right) \\
& \leq C\left(\left\|u-P_{h} u\right\|_{1}+\left\|\eta_{t}\right\|_{1}\right)\|\theta\|_{1}
\end{aligned}
$$

which implies that by Theorem 3.1 and (3.7)

$$
\begin{aligned}
\|\theta\|_{1} & \leq C\left(\left\|u-P_{h} u\right\|_{1}+\left\|\eta_{t}\right\|_{1}\right) \\
& \leq C\left(\frac{h^{\mu-1}}{r^{s-2}}\right)\left(\left\|u_{0}\right\|_{s}+\|u\|_{s}+\left\|u_{t}\right\|_{s}+\left\|u_{t}\right\|_{L^{2}\left(H^{s}\right)}\right) .
\end{aligned}
$$

By (3.2) we get

$$
\begin{aligned}
B_{\lambda}\left(u ; \theta_{t}, \theta_{t}\right) & =B_{\lambda}\left(u ; u_{t}+P_{h} u_{t}-u_{t}-\widetilde{u}_{t}, \theta_{t}\right) \\
& =B_{\lambda}\left(u ; \eta_{t}, \theta_{t}\right)-B_{\lambda}\left(u ; u_{t}-P_{h} u_{t}, \theta_{t}\right) \\
& =-A_{\lambda}\left(u ; \eta, \theta_{t}\right)-B_{\lambda}\left(u ; u_{t}-P_{h} u_{t}, \theta_{t}\right) .
\end{aligned}
$$

By Lemma 3.4 and Lemma 3.2, we have

$$
\left\|\theta_{t}\right\|_{1} \leq c\left(\|\eta\|_{1}+\left\|u_{t}-P_{h} u_{t}\right\|\right) \leq C \frac{h^{\mu-1}}{r^{s-2}}\left(\left\|u_{0}\right\|_{s}+\left\|u_{t}\right\|_{s}+\left\|u_{t}\right\|_{L^{2}\left(H^{s}\right)}\right) .
$$

Lemma 4.2. For $a \lambda>0$ and $\beta=1 /(d-1)$ there exists a constant $C$ satisfying if $u_{0} \in H^{s}, u(t) \in H^{s}, u(t) \in W^{1, \infty}$ and $u_{t} \in L^{2}\left(H^{s}\right)$, then

(i) $\|\nabla \widetilde{u}\|_{L^{\infty}} \leq C\left(\left\|u_{0}\right\|_{s}+\|u\|_{s}+\|u\|_{1, \infty}+\left\|u_{t}\right\|_{L^{2}\left(H^{s}\right)}\right)$,

(ii) $\|\nabla \widetilde{u}\|_{L^{\infty}\left(e_{k}\right)} \leq C\left(\left\|u_{0}\right\|_{s}+\|u\|_{s}+\|u\|_{1, \infty}+\left\|u_{t}\right\|_{s}+\left\|u_{t}\right\|_{L^{2}\left(H^{s}\right)}\right)$ 
hold and if $u_{0} \in H^{s}, u_{t} \in L^{2}\left(H^{s}\right)$ and $u_{t}(t) \in W^{1, \infty}$, then

(iii) $\left\|\nabla \widetilde{u}_{t}\right\|_{L^{\infty}} \leq C\left(\left\|u_{0}\right\|_{s}+\left\|u_{t}\right\|_{s}+\left\|u_{t}\right\|_{1, \infty}+\left\|u_{t}\right\|_{L^{2}\left(H^{s}\right)}\right)$,

(iv) $\left\|\nabla \widetilde{u}_{t}\right\|_{L^{\infty}\left(e_{k}\right)} \leq C\left(\left\|u_{0}\right\|_{s}+\left\|u_{t}\right\|_{s}+\left\|u_{t}\right\|_{1, \infty}+\left\|u_{t}\right\|_{L^{2}\left(H^{s}\right)}\right)$

hold, where $\mu=\min (r+1, s), s \geq \frac{d}{2}+1$ and $r \geq d / 2$.

Proof. By Lemma 3.2 and Theorem 3.1, we note that

$$
\begin{aligned}
\|\nabla \widetilde{u}\|_{L^{\infty}} & \leq\left\|\nabla\left(\widetilde{u}-P_{h} u\right)\right\|_{L^{\infty}}+\left\|\nabla\left(P_{h} u\right)-\nabla u\right\|_{L^{\infty}}+\|\nabla u\|_{L^{\infty}} \\
& \leq C\left(h^{-\frac{d}{2}}\left\|\nabla\left(\widetilde{u}-P_{h} u\right)\right\|_{L^{2}}+\|u\|_{1, \infty}\right) \\
& \leq C h^{-\frac{d}{2}}\left(\|\nabla \widetilde{u}-\nabla u\|_{L^{2}}+\left\|\nabla u-\nabla P_{h} u\right\|_{L^{2}}\right)+C\|u\|_{1, \infty} \\
& \leq C h^{-\frac{d}{2}} \frac{h^{\mu-1}}{r^{s-2}}\left(\left\|u_{0}\right\|_{s}+\|u\|_{s}+\left\|u_{t}\right\|_{L^{2}\left(H^{s}\right)}\right)+C\|u\|_{1, \infty} \\
& \leq C\left(\left\|u_{0}\right\|_{s}+\|u\|_{s}+\|u\|_{1, \infty}+\left\|u_{t}\right\|_{L^{2}\left(H^{s}\right)}\right)
\end{aligned}
$$

which completes the proof of (i). If $e_{k}=E_{i} \cap E_{j}=E_{i j}$, by Lemma 3.2, Lemma 3.1 and Lemma 4.1, we have

$$
\begin{aligned}
& \|\nabla \widetilde{u}\|_{L^{\infty}\left(e_{k}\right)} \\
\leq & \left\|\nabla\left(\widetilde{u}-P_{h} u\right)\right\|_{L^{\infty}\left(e_{k}\right)}+\left\|\nabla P_{h} u\right\|_{L^{\infty}\left(e_{k}\right)} \\
\leq & C h^{-\frac{(d-1)}{2}}\left\|\nabla\left(\widetilde{u}-P_{h} u\right)\right\|_{L^{2}\left(e_{k}\right)}+C\|\nabla u\|_{L^{\infty}\left(E_{i} \cup E_{j}\right)} \\
\leq & C h^{-\frac{(d-1)}{2}} h^{-\frac{1}{2}}\left(\left\|\nabla\left(\widetilde{u}-P_{h} u\right)\right\|_{L^{2}\left(E_{i} \cup E_{j}\right)}+h\left\|\nabla^{2}\left(\widetilde{u}-P_{h} u\right)\right\|_{L^{2}\left(E_{i} \cup E_{j}\right)}\right) \\
& +C\|u\|_{1, \infty} \\
\leq & C h^{-\frac{d}{2}} \frac{h^{\mu-1}}{r^{s-2}}\left(\left\|u_{0}\right\|_{s}+\|u\|_{s}+\left\|u_{t}\right\|_{s}+\left\|u_{t}\right\|_{L^{2}\left(H^{s}\right)}\right)+C\|u\|_{1, \infty} .
\end{aligned}
$$

This proves (ii) since $\mu \geq d / 2+1$. By the similar method, (iii) and (iv) can be proved.

Throughout this paper $\varepsilon, \varepsilon_{1}$ and $\varepsilon_{2}$ denote generic positive constants sufficiently small depending on $C$ and the Sobolev norms of $u$ but independent of $h$ and $r$.

Remark. By Lemma 3.2, $\|u(x, 0)-U(x, 0)\|_{L^{\infty}}=\left\|u_{0}(x)-\widetilde{u}(x, 0)\right\|_{L^{\infty}}=$ $\left\|u_{0}(x)-P_{h}\left(u_{0}(x)\right)\right\|_{L^{\infty}}<K^{*}$ holds.

Lemma 4.3. For a sufficiently small $\varepsilon>0$ and a generic constant $C>0$ the following estimation holds:

$$
\begin{aligned}
& \mid \sum_{k=1}^{L_{h}} \int_{e_{k}}\left\{(a(x, U)+b(x, U)) \nabla \xi \cdot \boldsymbol{n}_{\boldsymbol{k}}\right\}\left[\xi_{t}\right] d s \\
& \quad+\sum_{k=1}^{L_{h}} \int_{e_{k}}\left\{(a(x, U)+b(x, U)) \nabla \xi_{t} \cdot \boldsymbol{n}_{\boldsymbol{k}}\right\}[\xi] d s \mid \\
& \left.\leq \varepsilon\left\|\xi_{t}\right\|_{1}^{2}+C((a(x, U)+b(x, U)) \nabla \xi, \nabla \xi)+J_{\beta}^{\sigma}(\xi, \xi)\right) .
\end{aligned}
$$


Proof. Since $a(x, y)$ and $b(x, y)$ satisfy Condition (A), we have

$$
\begin{aligned}
& \quad \mid \sum_{k=1}^{L_{h}} \int_{e_{k}}\left\{(a(x, U)+b(x, U)) \nabla \xi \cdot \boldsymbol{n}_{\boldsymbol{k}}\right\}\left[\xi_{t}\right] d s \\
& \quad+\sum_{k=1}^{L_{h}} \int_{e_{k}}\left\{(a(x, U)+b(x, U)) \nabla \xi_{t} \cdot \boldsymbol{n}_{\boldsymbol{k}}\right\}[\xi] d s \mid \\
& \leq C \sum_{k=1}^{L_{h}}\left(\|\nabla \xi\|_{L^{2}\left(e_{k}\right)}\left\|\left[\xi_{t}\right]\right\|_{L^{2}\left(e_{k}\right)}+\left\|\nabla \xi_{t}\right\|_{L^{2}\left(e_{k}\right)}\|[\xi]\|_{L^{2}\left(e_{k}\right)}\right) \\
& \leq C\left(h^{(d-1) \beta / 2} \sum_{k=1}^{L_{h}}\|\nabla \xi\|_{L^{2}\left(e_{k}\right)} \frac{1}{\left|e_{k}\right|^{\beta / 2}}\left\|\left[\xi_{t}\right]\right\|_{L^{2}\left(e_{k}\right)}\right. \\
& \left.\quad+h^{(d-1) \beta / 2} \sum_{k=1}^{L_{h}}\left\|\nabla \xi_{t}\right\|_{L^{2}\left(e_{k}\right)} \frac{1}{\left|e_{k}\right|^{\beta / 2}}\|[\xi]\|_{L^{2}\left(e_{k}\right)}\right) \\
& \leq C h \sum_{k=1}^{L_{h}}\|\nabla \xi\|_{L^{2}\left(e_{k}\right)}^{2}+\varepsilon_{1}\left\|\xi_{t}\right\|_{1}^{2}+\varepsilon_{1} h \sum_{k=1}^{L_{h}}\left\|\nabla \xi_{t}\right\|_{L^{2}\left(e_{k}\right)}^{2}+C J_{\beta}^{\sigma}(\xi, \xi) \\
& \left.<\varepsilon\left\|\xi_{t}\right\|_{1}^{2}+C((a(x, U)+b(x, U)) \nabla \xi, \nabla \xi)+J_{\beta}^{\sigma}(\xi, \xi)\right)
\end{aligned}
$$

where we apply Lemma 3.1 and Condition (A).

Lemma 4.4. For a sufficiently small $\varepsilon>0$, if $u_{0} \in H^{s}, u(t) \in H^{s}, u(t) \in$ $W^{1, \infty}, u_{t} \in L^{2}\left(H^{s}\right), u_{t}(t) \in W^{1, \infty}$ we have the following estimations:

(i) $\left|A_{\lambda}\left(u ; \widetilde{u}, \xi+\xi_{t}\right)-A_{\lambda}\left(U ; \widetilde{u}, \xi+\xi_{t}\right)\right|$ $\leq C\left(\|\eta\|^{2}+h^{2}\|\nabla \eta\|^{2}+\|\xi\|^{2}\right)+3 \varepsilon\|\xi\|_{1}^{2}+3 \varepsilon\left\|\xi_{t}\right\|_{1}^{2}$

(ii) $\left|B_{\lambda}\left(u ; \widetilde{u}_{t}, \xi+\xi_{t}\right)-B_{\lambda}\left(U ; \widetilde{u}_{t}, \xi+\xi_{t}\right)\right|$

$$
\leq C\left(\|\eta\|^{2}+h^{2}\|\nabla \eta\|^{2}+\|\xi\|^{2}\right)+3 \varepsilon\|\xi\|_{1}^{2}+3 \varepsilon\left\|\xi_{t}\right\|_{1}^{2} .
$$

Proof. By the definitions of $A_{\lambda}, A_{\lambda}\left(U ; \widetilde{u}, \xi+\xi_{t}\right)-A_{\lambda}\left(u ; \widetilde{u}, \xi+\xi_{t}\right)$ can be separated as follows,

$$
\begin{aligned}
& A_{\lambda}\left(U ; \widetilde{u}, \xi+\xi_{t}\right)-A_{\lambda}\left(u ; \widetilde{u}, \xi+\xi_{t}\right) \\
= & \left((a(x, U)-a(x, u)) \nabla \widetilde{u}, \nabla\left(\xi+\xi_{t}\right)\right) \\
& -\sum_{k=1}^{L_{h}} \int_{e_{k}}\left\{(a(x, U)-a(x, u)) \nabla \widetilde{u} \cdot \boldsymbol{n}_{\boldsymbol{k}}\right\}\left[\xi+\xi_{t}\right] d s \\
& -\sum_{k=1}^{L_{h}} \int_{e_{k}}\left\{(a(x, U)-a(x, u)) \nabla\left(\xi+\xi_{t}\right) \cdot \boldsymbol{n}_{\boldsymbol{k}}\right\}[\widetilde{u}] d s \\
= & E_{1}+E_{2}+E_{3} .
\end{aligned}
$$

Now by applying Lemma 4.2, we get

$$
\left|E_{1}\right|=\left|\left((a(x, U)-a(x, u)) \nabla \widetilde{u}, \nabla\left(\xi+\xi_{t}\right)\right)\right|
$$




$$
\begin{aligned}
& \leq C\|\nabla \widetilde{u}\|_{L^{\infty}}\|u-U\|\left\|\nabla \xi+\nabla \xi_{t}\right\| \\
& \leq C(\|\eta\|+\|\xi\|)\left(\|\nabla \xi\|+\left\|\nabla \xi_{t}\right\|\right) \\
& \leq C\left(\|\eta\|^{2}+\|\xi\|^{2}\right)+\varepsilon\|\xi\|_{1}^{2}+\varepsilon\left\|\xi_{t}\right\|_{1}^{2} .
\end{aligned}
$$

Applying Lemma 4.2 and the trace inequalities yields

$$
\begin{aligned}
\left|E_{2}\right|=\left|\sum_{k=1}^{L_{h}} \int_{e_{k}}\left\{(a(x, U)-a(x, u)) \nabla \widetilde{u} \cdot \boldsymbol{n}_{\boldsymbol{k}}\right\}\left[\xi+\xi_{t}\right] d s\right| \\
\leq C \sum_{k=1}^{L_{h}}\|\nabla \widetilde{u}\|_{L^{\infty}\left(e_{k}\right)}\|u-U\|_{L^{2}\left(e_{k}\right)}\left(\|[\xi]\|_{L^{2}\left(e_{k}\right)}+\left\|\left[\xi_{t}\right]\right\|_{L^{2}\left(e_{k}\right)}\right) \\
\leq C \sum_{k=1}^{L_{h}}\|\nabla \widetilde{u}\|_{L^{\infty}\left(e_{k}\right)}\left(\|\eta\|_{L^{2}\left(e_{k}\right)}+\|\xi\|_{L^{2}\left(e_{k}\right)}\right) h^{\frac{(d-1) \beta}{2}}\left(\frac{1}{\left.\left|e_{k}\right|\right|^{\beta / 2}}\|[\xi]\|_{L^{2}\left(e_{k}\right)}\right. \\
\left.\quad+\frac{1}{\left|e_{k}\right|^{\beta / 2}}\left\|\left[\xi_{t}\right]\right\|_{L^{2}\left(e_{k}\right)}\right) \\
\leq C\left(\|\eta\|^{2}+h^{2}\|\nabla \eta\|^{2}+\|\xi\|^{2}\right)+\varepsilon\|\xi\|_{1}^{2}+\varepsilon\left\|\xi_{t}\right\|_{1}^{2} .
\end{aligned}
$$

By applying Lemma 3.1 and Theorem 3.1

$$
\begin{aligned}
& \left|E_{3}\right| \\
= & \left|\sum_{k=1}^{L_{h}} \int_{e_{k}}\left\{(a(x, U)-a(x, u)) \nabla\left(\xi+\xi_{t}\right) \cdot \boldsymbol{n}_{\boldsymbol{k}}\right\}[\widetilde{u}] d s\right| \\
\leq & \sum_{k=1}^{L_{h}} \int_{e_{k}}\left|\left\{(a(x, U)-a(x, u)) \nabla\left(\xi+\xi_{t}\right) \cdot \boldsymbol{n}_{\boldsymbol{k}}\right\}[\eta]\right| d s \\
\leq & C \sum_{k=1}^{L_{h}}\left(\|\nabla \xi\|_{L^{\infty}\left(e_{k}\right)}+\left\|\nabla \xi_{t}\right\|_{L^{\infty}\left(e_{k}\right)}\right)\|\{u-U\}\|_{L^{2}\left(e_{k}\right)}\|[\eta]\|_{L^{2}\left(e_{k}\right)} \\
\leq & C \sum_{j=1}^{N_{h}} h^{-\frac{1}{2}}\left(\|\nabla \xi\|_{L^{2}\left(E_{j}\right)}+\left\|\nabla \xi_{t}\right\|_{L^{2}\left(E_{j}\right)}\right)\left(h^{-1 / 2}\|\eta\|_{L^{2}\left(E_{j}\right)}+h^{1 / 2}\|\nabla \eta\|_{L^{2}\left(E_{j}\right)}\right. \\
& \left.+h^{-1 / 2}\|\xi\|_{L^{2}\left(E_{j}\right)}\right) \cdot h^{-\frac{1}{2}}\left(\|\eta\|_{L^{2}\left(E_{j}\right)}+h\|\nabla \eta\|_{L^{2}\left(E_{j}\right)}\right) \\
\leq & C\left(\|\nabla \xi\|+\left\|\nabla \xi_{t}\right\|\right)(\|\eta\|+h\|\nabla \eta\|+\|\xi\|)\left(\left\|u_{t}\right\|_{L^{2}\left(H^{s}\right)}+\left\|u_{0}\right\|_{s}\right) \\
\leq & C\left(\|\eta\|^{2}+h^{2}\|\nabla \eta\|^{2}+\|\xi\|^{2}\right)+\varepsilon\|\xi\|_{1}^{2}+\varepsilon\left\|\xi_{t}\right\|_{1}^{2},
\end{aligned}
$$

where $C$ depends on $\left\|u_{t}\right\|_{L^{2}\left(H^{s}\right)}$ and $\left\|u_{0}\right\|_{s}$. Combining the estimations of $E_{1}$, $E_{2}$ and $E_{3}$ we have

$$
\begin{aligned}
& \mid A_{\lambda}\left(U ; \widetilde{u}, \xi+\xi_{t}\right)-A_{\lambda}\left(u ; \widetilde{u}, \xi+\xi_{t} \mid\right. \\
\leq & C\left(\|\eta\|^{2}+h^{2}\|\nabla \eta\|^{2}+\|\xi\|^{2}\right)+3 \varepsilon\|\xi\|_{1}^{2}+3 \varepsilon\left\|\xi_{t}\right\|_{1}^{2},
\end{aligned}
$$


which completes the proof of (i). Similarly, by applying Lemma 4.2(iii), (iv) we can obtain the estimation (ii) as follows

$$
\begin{aligned}
& \left|B_{\lambda}\left(u ; \widetilde{u}_{t}, \xi+\xi_{t}\right)-B_{\lambda}\left(U ; \widetilde{u}_{t}, \xi+\xi_{t}\right)\right| \\
\leq & C\left(\|\eta\|^{2}+h^{2}\|\nabla \eta\|^{2}+\|\xi\|^{2}\right)+3 \varepsilon\|\xi\|_{1}^{2}+3 \varepsilon\left\|\xi_{t}\right\|_{1}^{2},
\end{aligned}
$$

where $C$ depends on $\left\|u_{0}\right\|_{s},\left\|u_{t}\right\|_{s},\left\|u_{t}\right\|_{1, \infty}$ and $\left\|u_{t}\right\|_{L^{2}\left(H^{s}\right)}$.

Theorem 4.2. For $a \lambda>1$ and $\beta=\frac{1}{d-1}$, if $u_{0} \in H^{s}, u(t) \in H^{s}, u(t) \in$ $W^{1, \infty}, u_{t} \in L^{2}\left(H^{s}\right), u_{t}(t) \in W^{1, \infty}$ and $u_{t t} \in L^{2}\left(H^{s}\right)$ and $f$ is locally Lipschitz continuous at $u$, i.e., there exists $c\left(u, K^{*}\right)$ such that if $|u(x, t)-v| \leq 2 K^{*}$, then $|f(x, u(x, t))-f(x, v)| \leq c\left(u, K^{*}\right)|u(x, t)-v|, \forall(x, t) \in \Omega \times[0, T], \forall v \in \mathbb{R}$, then there exists a constant $C>0$ independent of $h$ and $r$ such that

$$
\|u(\cdot, t)-U(\cdot, t)\| \leq C \frac{h^{\mu}}{r^{s-2}}\left(\left\|u_{0}\right\|_{s}+\left\|u_{t}\right\|_{L^{2}\left(H^{s}\right)}+\left\|u_{t t}\right\|_{L^{\infty}\left(H^{s}\right)}\right),
$$

where $\mu=\min (r+1, s), r \geq \frac{d}{2}$ and $s \geq 1+\frac{d}{2}$.

Proof. From (3.1), (3.3) and the definitions of $A_{\lambda}$ and $B_{\lambda}$ we have

$$
\begin{aligned}
& \left(u_{t t}-\widetilde{u}_{t t}+\widetilde{u}_{t t}-U_{t t}, v\right)+A_{\lambda}(u ; u-\widetilde{u}, v)+A_{\lambda}(u ; \widetilde{u}, v) \\
& +A_{\lambda}(U ; \widetilde{u}-U, v)-A_{\lambda}(U ; \widetilde{u}, v)+B_{\lambda}\left(u ;\left(u_{t}-\widetilde{u}_{t}\right), v\right)+B_{\lambda}\left(u ; \widetilde{u}_{t}, v\right) \\
& +B_{\lambda}\left(U ; \widetilde{u}_{t}-U_{t}, v\right)-B_{\lambda}\left(U ; \widetilde{u}_{t}, v\right) \\
= & (f(x, u)-f(x, U), v)+\lambda(u-U, v)+\lambda\left(u_{t}-U_{t}, v\right), \quad \forall v \in D_{r}\left(\mathcal{E}_{h}\right) .
\end{aligned}
$$

Now, we choose $v=\xi+\xi_{t}$ as a test function in (4.3) and we apply (3.2) to get the following

$$
\begin{aligned}
& \left(\eta_{t t}+\xi_{t t}, \xi+\xi_{t}\right)+A_{\lambda}\left(u ; \widetilde{u}, \xi+\xi_{t}\right)+A_{\lambda}\left(U ; \xi, \xi+\xi_{t}\right)-A_{\lambda}\left(U ; \widetilde{u}, \xi+\xi_{t}\right) \\
& +B_{\lambda}\left(u ; \widetilde{u}_{t}, \xi+\xi_{t}\right)+B_{\lambda}\left(U ; \xi_{t}, \xi+\xi_{t}\right)-B_{\lambda}\left(U ; \widetilde{u}_{t}, \xi+\xi_{t}\right) \\
= & \left(f(x, u)-f(x, U), \xi+\xi_{t}\right)+\lambda\left(u-U, \xi+\xi_{t}\right)+\lambda\left(u_{t}-U_{t}, \xi+\xi_{t}\right) .
\end{aligned}
$$

By simple computation we get

$$
\begin{aligned}
& \left(\xi_{t t}, \xi_{t}\right)+\left(\xi_{t t}, \xi\right)+A_{\lambda}(U ; \xi, \xi)+B_{\lambda}\left(U ; \xi_{t}, \xi_{t}\right)+A_{\lambda}\left(U ; \xi, \xi_{t}\right)+B_{\lambda}\left(U ; \xi_{t}, \xi\right) \\
= & -A_{\lambda}\left(u ; \widetilde{u}, \xi+\xi_{t}\right)+A_{\lambda}\left(U ; \widetilde{u}, \xi+\xi_{t}\right)-B_{\lambda}\left(u ; \widetilde{u}_{t}, \xi+\xi_{t}\right)+B_{\lambda}\left(U ; \widetilde{u}_{t}, \xi+\xi_{t}\right) \\
& +\left(f(x, u)-f(x, U), \xi+\xi_{t}\right)+\lambda\left(u-U, \xi+\xi_{t}\right)+\lambda\left(u_{t}-U_{t}, \xi+\xi_{t}\right) \\
& -\left(\eta_{t t}, \xi+\xi_{t}\right) .
\end{aligned}
$$

Applying the definitions of $A_{\lambda}\left(U ; \xi, \xi_{t}\right)$ and $B_{\lambda}\left(U ; \xi_{t}, \xi\right)$ in (4.4) we obtain

$$
\begin{aligned}
& \left(\xi_{t t}, \xi_{t}\right)+A_{\lambda}(U ; \xi, \xi)+B_{\lambda}\left(U ; \xi_{t}, \xi_{t}\right)+\left(\xi_{t t}, \xi\right) \\
& +\frac{1}{2} \frac{d}{d t}\left((a(x, U) \nabla \xi, \nabla \xi)+\lambda(\xi, \xi)+J_{\beta}^{\sigma}(\xi, \xi)\right) \\
& +\frac{1}{2} \frac{d}{d t}\left((b(x, U) \nabla \xi, \nabla \xi)+\lambda(\xi, \xi)+J_{\beta}^{\sigma}(\xi, \xi)\right)
\end{aligned}
$$




$$
\begin{aligned}
= & \frac{1}{2}\left(\frac{\partial}{\partial t}(a(x, U)+b(x, U)) \nabla \xi, \nabla \xi\right) \\
& +\sum_{k=1}^{L_{h}} \int_{e_{k}}\left\{(a(x, U)+b(x, U)) \nabla \xi \cdot \boldsymbol{n}_{\boldsymbol{k}}\right\}\left[\xi_{t}\right] d s \\
& +\sum_{k=1}^{L_{h}} \int_{e_{k}}\left\{(a(x, U)+b(x, U)) \nabla \xi_{t} \cdot \boldsymbol{n}_{\boldsymbol{k}}\right\}[\xi] d s \\
& -A_{\lambda}\left(u ; \widetilde{u}, \xi+\xi_{t}\right)+A_{\lambda}\left(U ; \widetilde{u}, \xi+\xi_{t}\right)-B_{\lambda}\left(u ; \widetilde{u}_{t}, \xi+\xi_{t}\right)+B_{\lambda}\left(U ; \widetilde{u}_{t}, \xi+\xi_{t}\right) \\
& +\left(f(x, u)-f(x, U), \xi+\xi_{t}\right)+\lambda\left(u-U, \xi+\xi_{t}\right)+\lambda\left(u_{t}-U_{t}, \xi+\xi_{t}\right) \\
& -\left(\eta_{t t}, \xi+\xi_{t}\right) .
\end{aligned}
$$

By applying Lemma 3.4 to the above equation, we have

$$
\begin{aligned}
& \frac{1}{2} \frac{d}{d t}\left\|\xi_{t}\right\|^{2}+\underset{\sim}{c}\|\xi\|_{1}^{2}+\underset{\sim}{c}\left\|\xi_{t}\right\|_{1}^{2} \\
& +\frac{d}{d t}\left(\frac{1}{2}((a(x, U)+b(x, U)) \nabla \xi, \nabla \xi)+\lambda(\xi, \xi)+J_{\beta}^{\sigma}(\xi, \xi)\right) \\
\leq & -\left(\xi_{t t}, \xi\right)-\left(\eta_{t t}, \xi+\xi_{t}\right)+\frac{1}{2}\left(\frac{\partial}{\partial t}(a(x, U)+b(x, U)) \nabla \xi, \nabla \xi\right) \\
& +\left(\sum_{k=1}^{L_{h}} \int_{e_{k}}\left\{(a(x, U)+b(x, U)) \nabla \xi \cdot \boldsymbol{n}_{\boldsymbol{k}}\right\}\left[\xi_{t}\right] d s\right. \\
& \left.+\sum_{k=1}^{L_{h}} \int_{e_{k}}\left\{(a(x, U)+b(x, U)) \nabla \xi_{t} \cdot \boldsymbol{n}_{\boldsymbol{k}}\right\}[\xi] d s\right) \\
& +\left(-A_{\lambda}\left(u ; \widetilde{u}, \xi+\xi_{t}\right)+A_{\lambda}\left(U ; \widetilde{u}, \xi+\xi_{t}\right)\right) \\
& +\left(-B_{\lambda}\left(u ; \widetilde{u}_{t}, \xi+\xi_{t}\right)+B_{\lambda}\left(U ; \widetilde{u}_{t}, \xi+\xi_{t}\right)\right) \\
& +\left(f(x, u)-f(x, U), \xi+\xi_{t}\right)+\lambda\left(u-U, \xi+\xi_{t}\right) \\
& +\lambda\left(u_{t}-U_{t}, \xi+\xi_{t}\right) .
\end{aligned}
$$

To continue the proof we temporarily assume that there exists $0<h^{*}<1$ such that

$$
\left\|\xi_{t}(t)\right\|<\widetilde{C} \frac{h^{\frac{d}{2}+\delta_{0}}}{r^{s-2}}, \quad 0<\delta_{0}<1
$$

and

$$
\|\xi(t)\|_{L^{\infty}}<K^{*}
$$

hold for $h<h^{*}, \forall t \in[0, T]$ and $\widetilde{C}>0$. Later we verify that these hypotheses hold. By the hypothesis (4.6) and the inverse inequality we have

$$
\left\|\xi_{t}\right\|_{L^{\infty}} \leq C h^{-\frac{d}{2}}\left\|\xi_{t}\right\| \leq C h^{\delta_{0}}<C^{*}, \quad \forall t \in[0, T] .
$$


By the hypothesis (4.6), the inverse inequality, Lemma 3.1 and Lemma 4.1 we have

$$
\begin{aligned}
\|(u-U)(t)\|_{L^{\infty}} \leq & \left\|\left(u-P_{h} u\right)(t)\right\|_{L^{\infty}}+\left\|\left(P_{h} u-\widetilde{u}\right)(t)\right\|_{L^{\infty}}+\|(\widetilde{u}-U)(t)\|_{L^{\infty}} \\
\leq & C\left(h+\frac{h^{\mu-\frac{d}{2}}}{r^{s-2}}\right)\left(\|u\|_{W^{1, \infty}}+\left\|u_{0}\right\|_{s}+\|u\|_{s}+\left\|u_{t}\right\|_{s}\right. \\
& \left.\quad+\left\|u_{t}\right\|_{L^{2}\left(H^{s}\right)}\right)+K^{*} \\
\leq & 2 K^{*}
\end{aligned}
$$

Now we estimate the terms in the right hand side of (4.5). For sufficiently small $\varepsilon>0$, we have the following estimation for $\left(\eta_{t t}, \xi+\xi_{t}\right)$

$$
\left|\left(\eta_{t t}, \xi+\xi_{t}\right)\right| \leq\left\|\eta_{t t}\right\|\left(\|\xi\|+\left\|\xi_{t}\right\|\right) \leq C\|\xi\|^{2}+\varepsilon\left\|\xi_{t}\right\|^{2}+C\left\|\eta_{t t}\right\|^{2}
$$

By applying Condition (B) on the functions $a$ and $b$ and (4.8), we get the following estimation.

$$
\left|\frac{1}{2}\left(\frac{\partial}{\partial t}(a(x, U)+b(x, U)) \nabla \xi, \nabla \xi\right)\right| \leq C\left\|U_{t}\right\|_{L^{\infty}}\|\nabla \xi\|^{2} \leq C(a(x, U) \nabla \xi, \nabla \xi),
$$

where $C$ depends on $\|u\|_{L^{\infty}\left(L^{\infty}\right)}$ and $C^{*}$. By applying Lemma 4.3 we obtain

$$
\begin{aligned}
& \mid \sum_{k=1}^{L_{h}} \int_{e_{k}}\left\{(a(x, U)+b(x, U)) \nabla \xi \cdot \boldsymbol{n}_{\boldsymbol{k}}\right\}\left[\xi_{t}\right] d s \\
& \quad+\sum_{k=1}^{L_{h}} \int_{e_{k}}\left\{(a(x, U)+b(x, U)) \nabla \xi_{t} \cdot \boldsymbol{n}_{\boldsymbol{k}}\right\}[\xi] d s \mid \\
& \left.\leq \varepsilon\left\|\xi_{t}\right\|_{1}^{2}+C((a(x, U)+b(x, U)) \nabla \xi, \nabla \xi)+J_{\beta}^{\sigma}(\xi, \xi)\right) .
\end{aligned}
$$

By applying (4.8) and the local Lipschitz property of $f$ at $u(x, t)$, we obtain the estimations in the following,

$$
\begin{gathered}
\left|\left(f(x, u)-f(x, U), \xi+\xi_{t}\right)\right| \leq c\left(u, K^{*}\right)(\|\eta\|+\|\xi\|)\left(\|\xi\|+\left\|\xi_{t}\right\|\right) \\
\leq C\left(\|\eta\|^{2}+\|\xi\|^{2}\right)+\varepsilon\left\|\xi_{t}\right\|^{2} \\
\left|\lambda\left(u-U, \xi+\xi_{t}\right)\right| \leq \lambda(\|\eta\|+\|\xi\|)\left(\|\xi\|+\left\|\xi_{t}\right\|\right) \leq C\left(\|\eta\|^{2}+\|\xi\|^{2}\right)+\varepsilon\left\|\xi_{t}\right\|^{2}, \\
\left|\lambda\left(u_{t}-U_{t}, \xi+\xi_{t}\right)\right| \leq \lambda\left(\left\|\eta_{t}\right\|+\left\|\xi_{t}\right\|\right)\left(\|\xi\|+\left\|\xi_{t}\right\|\right) \\
\leq C\left(\left\|\eta_{t}\right\|^{2}+\|\xi\|^{2}\right)+(\lambda+\varepsilon)\left\|\xi_{t}\right\|^{2} .
\end{gathered}
$$

Now substituting the above estimations and applying Lemma 4.3 and Lemma 4.4 in (4.5) we get

$$
\begin{aligned}
& \frac{d}{d t}\left(\frac{1}{2}\left\|\xi_{t}\right\|^{2}+\frac{1}{2}((a(x, U)+b(x, U)) \nabla \xi, \nabla \xi)+\lambda(\xi, \xi)+J_{\beta}^{\sigma}(\xi, \xi)\right)+\stackrel{c}{2}\|\xi\|_{1}^{2} \\
& +\frac{\widetilde{c}}{2}\left\|\xi_{t}\right\|_{1}^{2} \\
\leq & -\left(\xi_{t t}, \xi\right)+C\left(\left\|\eta_{t t}\right\|^{2}+((a(x, U)+b(x, U)) \nabla \xi, \nabla \xi)+J_{\beta}^{\sigma}(\xi, \xi)\right.
\end{aligned}
$$




$$
\left.+\|\eta\|^{2}+\left\|\eta_{t}\right\|^{2}+h^{2}\|\nabla \eta\|^{2}+\|\xi\|^{2}+\left\|\xi_{t}\right\|^{2}\right) .
$$

Applying the integration by parts and Cauchy Schwarz's inequality we have

$$
\begin{aligned}
-\int_{0}^{t}\left(\xi_{t t}, \xi\right) d \tau \leq & \frac{1}{4}\left\|\xi_{t}(t)\right\|^{2}+\|\xi(t)\|^{2}+\frac{1}{2}\left\|\xi_{t}(0)\right\|^{2}+\frac{1}{2}\|\xi(0)\|^{2} \\
& +\int_{0}^{t}\left\|\xi_{t}\right\|^{2} d \tau .
\end{aligned}
$$

By integrating both sides of (4.9) from 0 to $t$ and applying (4.10), we have

$$
\begin{aligned}
& \frac{1}{2}\left\|\xi_{t}(t)\right\|^{2}+\frac{1}{2}((a(x, U)+b(x, U)) \nabla \xi(t), \nabla \xi(t))+\lambda\|\xi(t)\|^{2}+J_{\beta}^{\sigma}(\xi(t), \xi(t)) \\
& +\frac{c}{2}\left(\int_{0}^{t}\|\xi\|_{1}^{2}+\left\|\xi_{t}\right\|_{1}^{2} d \tau\right) \\
\leq & \frac{1}{2}\left\|\xi_{t}(0)\right\|^{2}+\frac{1}{2}((a(x, U)+b(x, U)) \nabla \xi(0), \nabla \xi(0))+\lambda\|\xi(0)\|^{2}+J_{\beta}^{\sigma}(\xi(0), \xi(0)) \\
& +\frac{1}{4}\left\|\xi_{t}(t)\right\|^{2}+\|\xi(t)\|^{2}+\frac{1}{2}\left\|\xi_{t}(0)\right\|^{2}+\frac{1}{2}\|\xi(0)\|^{2} \\
& +C\left(\int_{0}^{t}\left(\left\|\eta_{t t}\right\|^{2}+\left\|\eta_{t}\right\|^{2}+\|\eta\|^{2}+h^{2}\|\nabla \eta\|^{2}\right) d \tau\right) \\
& +C\left(\int_{0}^{t}\left(((a(U)+b(U)) \nabla \xi, \nabla \xi)+J_{\beta}^{\sigma}(\xi, \xi)+\|\xi\|^{2}+\left\|\xi_{t}\right\|^{2}\right) d \tau\right) .
\end{aligned}
$$

Adopting the initial approximations from (3.3) to the inequality above, we obtain

$$
\begin{aligned}
& \frac{1}{4}\left\|\xi_{t}(t)\right\|^{2}+\frac{1}{2}((a(x, U)+b(x, U)) \nabla \xi(t), \nabla \xi(t))+(\lambda-1)\|\xi(t)\|^{2} \\
& +J_{\beta}^{\sigma}(\xi(t), \xi(t))+\frac{c}{2} \int_{0}^{t}\left(\|\xi\|_{1}^{2}+\left\|\xi_{t}\right\|_{1}^{2}\right) d \tau \\
\leq & C \int_{0}^{t}\left\|\xi_{t}\right\|^{2} d \tau+C\left(\int_{0}^{t}\left(\|\eta\|^{2}+h^{2}\|\nabla \eta\|^{2}+\left\|\eta_{t}\right\|^{2}+\left\|\eta_{t t}\right\|^{2}\right) d \tau\right) \\
& +C\left(\int_{0}^{t}\left(((a(x, U)+b(x, U)) \nabla \xi, \nabla \xi)+J_{\beta}^{\sigma}(\xi, \xi)+\|\xi\|^{2} d \tau\right)\right.
\end{aligned}
$$

for $\lambda>1$. By applying the Gronwall's Lemma, we have

$$
\begin{aligned}
& \left\|\xi_{t}(t)\right\|^{2}+((a(x, U)+b(x, U)) \nabla \xi(t), \nabla \xi(t))+\|\xi(t)\|^{2}+J_{\beta}^{\sigma}(\xi(t), \xi(t)) \\
\leq & C \int_{0}^{t}\left(\|\eta\|^{2}+h^{2}\|\nabla \eta\|^{2}+\left\|\eta_{t}\right\|^{2}+\left\|\eta_{t t}\right\|^{2}\right) d \tau .
\end{aligned}
$$

Applying the estimations from Theorems 3.1 and 3.2 to the above inequality we get

$\left\|\xi_{t}(t)\right\|^{2}+\|\xi(t)\|^{2}+J_{\beta}^{\sigma}(\xi(t), \xi(t)) \leq C\left(\frac{h^{\mu}}{r^{s-2}}\right)^{2}\left(\left\|u_{0}\right\|_{s}^{2}+\left\|u_{t}\right\|_{L^{2}\left(H^{s}\right)}^{2}+\left\|u_{t t}\right\|_{L^{2}\left(H^{s}\right)}^{2}\right)$. 
Combining (4.11) and Theorem 3.1 we conclude that the following optimal error estimate holds

$$
\|u(\cdot, t)-U(\cdot, t)\| \leq C \frac{h^{\mu}}{r^{s-2}}\left(\left\|u_{0}\right\|_{s}+\left\|u_{t}\right\|_{L^{2}\left(H^{s}\right)}+\left\|u_{t t}\right\|_{L^{2}\left(H^{s}\right)}\right),
$$

where $\mu=\min (r+1, s)$.

Now we show that the hypotheses (4.6) hold. From (3.3), (4.6) obviously holds for $t=0$. Now we assume that there exists $0<t^{*} \leq T$ such that (4.6) holds for $0 \leq t<t^{*}$ but

$$
\left\|\xi_{t}\left(t^{*}\right)\right\| \geq \widetilde{C} \frac{h^{\frac{d}{2}+\delta_{0}}}{r^{s-2}} .
$$

Now we take a sequence of $\left\{t_{n}\right\} \subset\left[0, t^{*}\right)$ such that $\lim _{n \rightarrow \infty} t_{n}=t^{*}$. For $t=$ $t_{n}$ the hypothesis (4.6) is satisfied and so (4.11) can be obtained for $t=t_{n}$ by the aforementioned process in this proof. Therefore $\left\|\xi_{t}\left(t_{n}\right)\right\| \leq C \frac{h^{\mu}}{r^{s-2}} \leq \frac{\widetilde{C}}{2} \frac{h^{\frac{d}{2}+\delta_{0}}}{r^{s-2}}$. By the continuity property of $\left\|\xi_{t}\right\|$ with respect to $t$ proved in Theorem 4.1 we get $\left\|\xi_{t}\left(t^{*}\right)\right\| \leq \frac{\widetilde{C}}{2} \frac{h^{\frac{d}{2}+\delta_{0}}}{r^{s-2}}$ which contradicts to (4.12). Therefore the hypothesis (4.6) holds. Now we will prove that the hypothesis (4.7) holds. Obviously (4.7) holds for $t=0$. Now we assume that there exists $\tau \in(0, T]$ such that $(4.7)$ holds for $0 \leq t<\tau$ but

$$
\|\xi(\tau)\|_{L^{\infty}} \geq K^{*}
$$

Now again we take a sequence of $\left\{t_{n}\right\} \subset[0, \tau)$ such that $\lim _{n \rightarrow \infty} t_{n}=\tau$. For $t=t_{n}$, by applying Lemma 3.2 and Lemma 4.1 we have

$$
\begin{aligned}
& \left\|(u-U)\left(t_{n}\right)\right\|_{L^{\infty}} \\
\leq & \left\|\left(u-P_{h} u\right)\left(t_{n}\right)\right\|_{L^{\infty}}+\left\|\left(P_{h} u-\widetilde{u}\right)\left(t_{n}\right)\right\|_{L^{\infty}}+\left\|\widetilde{u}\left(t_{n}\right)-U\left(t_{n}\right)\right\|_{L^{\infty}} \\
\leq & C h\|u\|_{1, \infty}+\left\|\theta\left(t_{n}\right)\right\|_{L^{\infty}}+\left\|\xi\left(t_{n}\right)\right\|_{L^{\infty}} \\
\leq & C\left(h+\frac{h^{\mu-\frac{d}{2}-1}}{r^{s-2}}\right)\left(\left\|u_{0}\right\|_{s}+\|u\|_{1, \infty}+\|u\|_{s}+\left\|u_{t}\right\|_{s}+\left\|u_{t}\right\|_{L^{2}\left(H^{2}\right)}\right)+K^{*},
\end{aligned}
$$

where $C$ depends on the sobolev norms of $u$. Therefore for sufficiently small $h$, we have $\left\|(u-U)\left(t_{n}\right)\right\|_{L^{\infty}} \leq 2 K^{*}$. For $t=t_{n}$ we conclude that the following property, needed to obtain the result $(4.11),\left|f(x, u(x, v))-f\left(x, U\left(x, t_{n}\right)\right)\right| \leq$ $c(u, K *)\left|u\left(x, t_{n}\right)-U\left(x, t_{n}\right)\right|$ holds. Therefore we have (4.11) holds for $t=t_{n}$

so that $\left\|\xi\left(t_{n}\right)\right\|_{L^{\infty}} \leq C \frac{h^{\mu-\frac{d}{2}}}{r^{s-2}}\left(\left\|u_{0}\right\|_{s}+\left\|u_{t}\right\|_{L^{2}\left(H^{s}\right)}+\left\|u_{t t}\right\|_{L^{2}\left(H^{s}\right)}\right) \leq \frac{K^{*}}{2}$ holds for sufficiently small $h$. In Theorem 4.1 we prove the continuity of $U(\mathrm{t})$ with respect to $t$ so we have the continuity of $\|\xi(t)\|_{L^{\infty}}$ hence we have $\|\xi(\tau)\|_{L^{\infty}} \leq \frac{K^{*}}{2}$ which contradicts to (4.13), so that the hypothesis (4.7) holds. This completes the proof of Theorem 4.2 .

\section{References}

[1] D. N. Arnold, An interior penalty finite element method with discontinuous elements, SIAM J. Numer. Anal. 19 (1982), no. 2, 724-760. 
[2] I. Babuška and M. Suri, The h-p version of the finite element method with quasi-uniform meshes, RAIRO Modél. Math. Anal. Numér. 21 (1987), no. 2, 199-238.

[3] _ The optimal convergence rates of the p-version of the finite element method, SIAM J. Numer. Anal. 24 (1987), no. 4, 750-776.

[4] B. Darlow, R. Ewing, and M. F. Wheeler, Mixed finite element methods for miscible displacement problems in porous media, Soc. Pet. Erg. Report SPE 10500, 1982.

[5] J. Douglas and T. Dupont, Interior penalty procedures for elliptic and parabolic Galerkin methods, Computing methods in applied sciences (Second Internat. Sympos., Versailles, 1975), pp. 207216. Lecture Notes in Phys., Vol. 58, Springer, Berlin, 1976.

[6] J. Douglas, M. F. Wheeler, B. L. Darlow, and R. P. Kendall, Self-adaptive finite element simulation of miscible displacement in porous media, Compuer Methods in Applied Mechanics and Engineering 47 (1984), 131-159.

[7] R. E. Ewing, Time-stepping Galerkin methods for nonlinear Sobolev partial differential equations, SIAM J. Numer. Anal. 15 (1978), no. 6, 1125-1150.

[8] H. Koch and S. Antman, Stability and Hopf bifurcation for fully nonlinear parabolic hyperbolic equations, SIAM J. Math. Anal. 32 (2000), no. 2, 360-384.

[9] A. Lasis and E. Suri, hp-version discontinuous Galerkin finite element method for semilinear parabolic problems, SIAM J. Math. Anal. 45 (2007), no. 4, 1544-1569

[10] Q. Lin and S. Zhang, A direct global superconvergence analysis for Sobolev and viscoelasticity type equations, Appl. Math. 42 (1997), no. 1, 23-34.

[11] J. A. Nitsche, Uber ein variationspringzip zur Losung von Dirichlet-problemen bei Verwendung von Teilraumen, die Keinen Randbedingungen underworfen sind, Abh. Math. Sem. Univ. Hamburg 36 (1971), 9-15.

[12] J. T. Oden, I. Babuška, and C. E. Baumann, A discontinuous hp finite element method for diffusion problems, J. Comput. Phys. 146 (1998), no. 2, 491-519.

[13] M. R. Ohm, H. Y. Lee, and J. Y. Shin, Error estimates for discontinuous Galerkin method for nonlinear parabolic equations, J. Math. Anal. Appl. 315 (2006), no. 1, 132143.

[14] B. Rivière and S. Shaw, Discontinuous Galerkin finite element approximation of nonlinear non-Fickian diffusion in viscoelastic polymers, SIAM J. Numer. Anal. 44 (2006), no. 6, 2650-2670.

[15] B. Rivière and M. F. Wheeler, A discontinuous Galerkin method applied to nonlinear parabolic equations, Discontinuous Galerkin methods (Newport, RI, 1999), 231-44, Lect. Notes Comput. Sci. Eng., 11, Springer, Berlin, 2000.

[16] S. Shaw and J. Whiteman, Numerical solution of linear quasistatic hereditary viscoelasticity problems, SIAM J. Numer. Anal. 38 (2000), no. 1, 80-97.

[17] M. F. Wheeler, An elliptic collocation finite element method with interior penalties, SIAM J. Numer. Anal. 15 (1978), no. 1, 152-161.

\section{Mi RAY OHM}

Division of INFORMATION Systems EngINEERING

DONGSEO UNIVERSITY

PUSAN 617-716, KOREA

E-mail address: mrohm@dongseo.ac.kr

Hyun Young LeE

Department of Mathematics

Kyungsung University

PUsAn 608-736, KoreA

E-mail address: hylee@ks.ac.kr 
Jun YONG SHIN

Department of Applied Mathematics

Pukyong National University

Pusan 608-737, Korea

E-mail address: jyshin@pknu.ac.kr 